\title{
Trigonometric Sutherland systems and their Ruijsenaars duals from symplectic reduction
}

\author{
L. Fehér ${ }^{a, b}$ and V. Ayadi ${ }^{b}$ \\ ${ }^{a}$ Department of Theoretical Physics, MTA KFKI RMKI \\ H-1525 Budapest, P.O.B. 49, Hungary \\ e-mail: lfeher@rmki.kfki.hu \\ ${ }^{b}$ Department of Theoretical Physics, University of Szeged \\ Tisza Lajos krt 84-86, H-6720 Szeged, Hungary \\ e-mail: ayadi.viktor@stud.u-szeged.hu
}

\begin{abstract}
Besides its usual interpretation as a system of $n$ indistinguishable particles moving on the circle, the trigonometric Sutherland system can be viewed alternatively as a system of distinguishable particles on the circle or on the line, and these 3 physically distinct systems are in duality with corresponding variants of the rational Ruijsenaars-Schneider system. We explain that the 3 duality relations, first obtained by Ruijsenaars in 1995, arise naturally from the Kazhdan-Kostant-Sternberg symplectic reductions of the cotangent bundles of the group $U(n)$ and its covering groups $U(1) \times S U(n)$ and $\mathbb{R} \times S U(n)$, respectively. This geometric interpretation enhances our understanding of the duality relations and simplifies Ruijsenaars' original direct arguments that led to their discovery.
\end{abstract}




\section{Introduction}

We deal here with certain aspects of integrable classical many-body systems in one spatial dimension, which is an important area of mathematical physics having many applications and connections to other areas as reviewed, for example, in [1, 2, 3, 4, 5].

In the impressive series of papers [6, 7, 8] Ruijsenaars established an intriguing duality relation among Calogero type integrable many-body systems. The phase spaces of the dual pairs of systems are related by a symplectomorphism that identifies the action variables of the 'first' system as the particle-positions of the 'second' system, and vice versa. The duality map, alias the action-angle transform, was constructed in [6, 7, 8] by direct means for each nonelliptic system associated with the $A_{n}$ root system, covering both the standard non-relativistic systems and their relativistic deformations [9]. It was used to analyze the classical dynamics of the systems, and later it was also shown to have a quantum mechanical counterpart, the so-called bispectral property [10, 11].

The self-dual character of the rational Calogero system was already noted by Kazhdan, Kostant and Sternberg (KKS) in their famous paper [12] that introduced the tool of symplectic reduction into the study of Calogero type systems. In fact, Ruijsenaars based his analysis on certain algebraic relations satisfied by the pertinent Lax matrices which are reminiscent of moment map constraints, and hinted that there might lurk a KKS type symplectic reduction picture behind the duality in general. This conjecture was later vigorously advocated in the work of Gorsky-Nekrasov and their collaborators [13, 14, 15, 16, 17, 18, 19], but it was not fully substantiated since the main concern of these authors was the reduction of infinite-dimensional phase spaces that pose serious technical difficulties. Their work contains interesting ideas also about finite-dimensional reductions related to duality. The elaboration of these ideas requires further effort since the topological subtleties and the distinctions between the complex phase spaces and their different real forms were swept under the carpet in the, otherwise quite remarkable, articles cited above.

One of us, jointly with C. Klimčík, recently explored the finite-dimensional symplectic reductions that explain the duality between the hyperbolic Sutherland and the rational RuijsenaarsSchneider systems [20] as well as the duality between two real forms of the trigonometric Ruijsenaars-Schneider system [21]. The present paper is devoted to other cases, namely, to the duality relations involving different variants of the trigonometric Sutherland system and their Ruijsenaars duals introduced originally in [8]. The ideas that we shall use here are similar to those applied in [20, 21], and basic observations were pointed out previously in [14, 16, 18. However, the details are quite non-trivial and each case needs a separate analysis. One cannot simply apply analytic continuation or degeneration of one case to the other, since there are considerable topological and analytical subtleties that cannot be handled by such methods.

It is necessary to recall that the trigonometric Sutherland system, formally given by the Hamiltonian

$$
H_{\text {Suth }}(q, p)=\frac{1}{2} \sum_{i=1}^{n} p_{i}^{2}+\frac{1}{4} \sum_{1 \leq i<j \leq n} \frac{x^{2}}{\sin ^{2}\left(\frac{q_{i}-q_{j}}{2}\right)},
$$

with real coupling parameter $x$, admits 3 physically different variants depending on the choice of the domain of the position variables [8. Specifically, one can view it most naturally as a system of $n$ indistinguishable particles moving on the circle, or as systems of distinguishable 
particles either on the circle or one the line. Respectively, the configuration spaces are chosen from the list

$$
Q(n), \quad U(1) \times S Q(n), \quad \mathbb{R} \times S Q(n),
$$

where $Q(n)$ belongs to indistinguishable particles, while in the latter two cases one can separate the freedom of center of mass motion either as $U(1) \simeq S^{1}$ or as $\mathbb{R}$, and $S Q(n)$ is the arena of the motion relative to the center of mass. In fact, $Q(n)$ can be realized as $Q(n) \equiv \mathbb{T}(n)^{0} / S(n)$, where $\mathbb{T}(n)^{0}$ is the regular part of the standard maximal torus of $U(n)$ on which the symmetric (Weyl) group $S(n)$ acts, and $S Q(n)$ is similarly related to the group $S U(n)$. The third configuration space in (1.2) is simply connected, and one has corresponding covering maps

$$
\mathbb{R} \times S Q(n) \longrightarrow U(1) \times S Q(n) \longrightarrow Q(n) .
$$

The action-angle transforms [8] of the alternative Sutherland phase spaces

$$
P:=T^{*} Q(n), \quad P_{1}:=T^{*} U(1) \times T^{*} S Q(n), \quad P_{2}:=T^{*} \mathbb{R} \times T^{*} S Q(n)
$$

are certain phase spaces

$$
\hat{P}_{c}, \quad \hat{P}_{1}:=T^{*} U(1) \times \mathbb{C}^{n-1}, \quad \hat{P}_{2}:=T^{*} \mathbb{R} \times \mathbb{C}^{n-1} .
$$

The structure of $\hat{P}_{c}$ and the symplectic forms are displayed in equations (3.67) and (4.33) below, but we note already here that $\hat{P}_{c}$ has the open dense submanifold $\hat{P}$ defined by

$$
\hat{P}=\mathbb{T}(n) \times \mathfrak{C}_{x}=\left\{\left(e^{\mathrm{i} \hat{q}}, \hat{p}\right)\right\}, \quad \mathfrak{C}_{x}:=\left\{\hat{p} \in \mathbb{R}^{n}\left|\hat{p}_{i}-\hat{p}_{i+1}>\right| x \mid, \quad i=1, \ldots, n-1\right\}
$$

equipped with the symplectic form $\hat{\omega}=\sum_{i=1}^{n} d \hat{p}_{i} \wedge d \hat{q}_{i}$ and the Hamiltonian

$$
\hat{H}_{\mathrm{RS}}(\hat{q}, \hat{p})=\sum_{a=1}^{n}\left(\cos \hat{q}_{a}\right) \prod_{k \neq a}\left[1-\frac{x^{2}}{\left(\hat{p}_{k}-\hat{p}_{a}\right)^{2}}\right]^{\frac{1}{2}}
$$

which is a particular real form of the complex rational Ruijsenaars-Schneider Hamiltonian [9]. The flows of the commuting family of Liouville integrable Hamiltonians that contains $\hat{H}_{\mathrm{RS}}$ are complete only on the full phase space $\hat{P}_{c}$.

It will be shown in the present work that the relations between the above phase spaces, described in [8] without reference to Lie groups, correspond to the covering homomorphisms

$$
G_{2}:=\mathbb{R} \times S U(n) \longrightarrow G_{1}:=U(1) \times S U(n) \longrightarrow G:=U(n) .
$$

For this, it will be used that the conjugation action of $G$ on $T^{*} G$ and the analogous actions of $G_{i}$ on $T^{*} G_{i}(i=1,2)$ represent actions of the same effective symmetry group

$$
\bar{G}:=G / \mathbb{Z}_{G} \simeq G_{1} / \mathbb{Z}_{G_{1}} \simeq G_{2} / \mathbb{Z}_{G_{2}}
$$

where $\mathbb{Z}_{G}$ stands for the center of $G$ and so on. By performing symplectic reductions at the usual KKS value of the moment map of the $\bar{G}$-action, we shall obtain covering Poisson maps between the respective reduced phase spaces,

$$
\left(T^{*} G_{2}\right)_{\mathrm{red}} \longrightarrow\left(T^{*} G_{1}\right)_{\mathrm{red}} \longrightarrow\left(T^{*} G\right)_{\mathrm{red}}
$$


from the homomorphisms (1.8). By constructing two alternative models (symplectomorphic images) of each of the 3 reduced phase spaces in (1.10), we shall arrive at the identifications

$$
P_{2} \simeq\left(T^{*} G_{2}\right)_{\mathrm{red}} \simeq \hat{P}_{2}, \quad P_{1} \simeq\left(T^{*} G_{1}\right)_{\mathrm{red}} \simeq \hat{P}_{1}, \quad P \simeq\left(T^{*} G\right)_{\mathrm{red}} \simeq \hat{P}_{c} .
$$

This will allow us to interpret the 'duality symplectomorphisms'

$$
\mathcal{R}_{2}: P_{2} \longrightarrow \hat{P}_{2}, \quad \mathcal{R}_{1}: P_{1} \longrightarrow \hat{P}_{1}, \quad \mathcal{R}: P \longrightarrow \hat{P}_{c}
$$

as the natural maps between the respective models of the same reduced phase space. By virtue of (1.10), then the following commutative diagram arises automatically from our considerations:

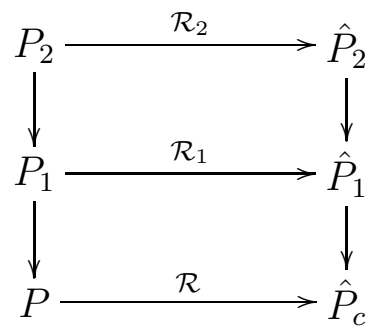

Here, the horizontal arrows represent duality symplectomorphims and the vertical arrows are covering (locally symplectic) Poisson maps. The maps corresponding to the arrows above have all been constructed originally by Ruijsenaars $[8]$, relying on rather demanding direct arguments. Our group theoretic interpretation throws a new light on the web of dualities and coverings encapsulated by the above diagram. This will also lead to technical simplifications in comparison to [8], concerning in particular the symplectic property of the map $\mathcal{R}$, which is obvious in our setting but was quite difficult to prove originally by direct methods.

So far we have not fully specified the commuting Hamiltonians of the dual pairs of systems, but it will be explained in the main text that they arise from two standard families of 'free' Hamiltonians on $T^{*} G$, and from their pullbacks to $T^{*} G_{i}$, via the KKS reduction. As for the logical organization of our arguments, we shall first describe the most complicated map $\mathcal{R}: P \rightarrow \hat{P}_{c}$ in detail, and then explain how the other maps in the diagram correspond to the covering homomorphisms (1.8).

In Section 2 we fix our notations and define the systems that we reduce subsequently. We describe the two models $P$ and $\hat{P}_{c}$ of the reduced phase space $\left(T^{*} G\right)_{\text {red }}$ in Section 3 , and derive also the dual pairs of Lax matrices that generate the commuting reduced Hamiltonians. The structure of the maps in the diagram (1.13) is explained in Section 4. Section 5 contains a brief summary together with a discussion of open problems. An appendix is included, where we characterize the three alternative phase spaces (1.4) of 'non-coinciding point-particles' moving on the line or on the circle.

\section{Setting the stage for symplectic reduction}

Consider the Lie group $G=U(n)$ and identify its Lie algebra $\mathcal{G}:=u(n)$ with the dual space $\mathcal{G}^{*}$ by means of the invariant scalar product

$$
\langle X, Y\rangle:=-\operatorname{tr}(X Y), \quad \forall X, Y \in \mathcal{G},
$$

\footnotetext{
${ }^{1}$ To facilitate comparison, we note that our symbols $P, P_{2}, \hat{P}_{c}, \hat{P}_{2}, \mathcal{R}, \mathcal{R}_{2}$ in (1.13) correspond respectively to $\Omega, \tilde{\Omega}, \hat{\Omega}^{\sharp}, \hat{\Omega}^{\sharp c}, \Phi^{\sharp}, \tilde{\Phi}^{\sharp}$ in diagram $(1.74)$ of [8], and our $\hat{P}$ in (1.6] corresponds to $\hat{\Omega}$ in $(1.67)$ of [8].
} 
and also use the identification

$$
T^{*} G \simeq G \times \mathcal{G}^{*} \simeq G \times \mathcal{G}=\{(g, J) \mid g \in G, J \in \mathcal{G}\}
$$

defined with the aid of right-translations on $G$. The cotangent bundle carries the symplectic form

$$
\Omega_{T^{*} G}=-d \operatorname{tr}\left(J d g g^{-1}\right) .
$$

The group $\bar{G}(1.9)$ can be realized as $\bar{G} \simeq S U(n) / \mathbb{Z}_{n}$ and this permits identification of its Lie algebra $\overline{\mathcal{G}}$ with $s u(n)$, which we regard also as the model of $\overline{\mathcal{G}}^{*}$ by taking advantage of the same invariant scalar product as in (2.1). We then let $\mathcal{O} \subset \overline{\mathcal{G}}^{*}$ denote the minimal coadjoint orbit of $\bar{G}$ provided by

$$
\mathcal{O}:=\left\{\xi=\left.\xi(x, v)\left|\xi(x, v):=\mathrm{i} x\left(\mathbf{1}_{n}-v v^{\dagger}\right), v \in \mathbb{C}^{n},\right| v\right|^{2}=n\right\},
$$

where $x$ is a non-zero real parameter. The Kirillov-Kostant-Souriau symplectic form $\Omega_{\mathcal{O}}$ of $\mathcal{O}$ can be written in terms of the redundant variables furnished by the components of the vector $v$ of length $\sqrt{n}$ as

$$
\Omega_{\mathcal{O}}=\mathrm{i} x d v^{\dagger} \wedge d v
$$

To clarify the meaning of formula (2.5), we note that the orbit $\mathcal{O}$ coincides with the projective space $\mathbb{C} P^{n-1}$ as a manifold, and we here view it as a reduction of the symplectic vector space $\mathbb{C}^{n} \simeq \mathbb{R}^{2 n}$ with respect to the natural action of $U(1)$ generated by the function $v \mapsto|v|^{2}$. By setting this $U(1)$ moment map equal to the constant $n$ and then factoring by $U(1)$, the symplectic form of $\mathbb{C}^{n}$, given by ixdv $\wedge d v$ but with unrestricted $v$, descends to the orbit $\mathcal{O}$. The resulting symplectic form on $\mathcal{O}$ is a multiple of the standard Fubini-Study form of $\mathbb{C} P^{n-1}$.

Our starting point for symplectic reduction will be the phase space $\left(M, \Omega_{M}\right)$ :

$$
M=T^{*} G \times \mathcal{O}, \quad \Omega_{M}=\Omega_{T^{*} G}+\Omega_{\mathcal{O}} .
$$

Corresponding to the symplectic form $\Omega_{M}$, the non-vanishing Poisson brackets between the matrix elements $g_{j k}$ and the components $J_{a}:=\left\langle J, T_{a}\right\rangle$ and $\xi_{a}:=\left\langle\xi, T_{a}\right\rangle$ are

$$
\left\{g_{j k}, J_{a}\right\}_{M}=\left(T_{a} g\right)_{j k}, \quad\left\{J_{a}, J_{b}\right\}_{M}=\left\langle J,\left[T_{a}, T_{b}\right]\right\rangle, \quad\left\{\xi_{a}, \xi_{b}\right\}_{M}=\left\langle\xi,\left[T_{a}, T_{b}\right]\right\rangle,
$$

where $\left\{T_{a}\right\}$ is an arbitrary basis of $\mathcal{G}$.

The phase space $M$ carries two families of 'free' Hamiltonians $\left\{\mathcal{H}_{k}\right\}$ and $\left\{\hat{\mathcal{H}}_{ \pm k}\right\}$ given by

$$
\mathcal{H}_{k}(g, J, \xi):=\frac{1}{k} \operatorname{tr}(-\mathrm{i} J)^{k}, \quad \forall k=1, \ldots, n,
$$

and

$$
\hat{\mathcal{H}}_{k}(g, J, \xi):=\frac{1}{k} \operatorname{tr}\left(g^{k}+g^{-k}\right), \quad \hat{\mathcal{H}}_{-k}(g, J, \xi):=\frac{1}{\mathrm{i} k} \operatorname{tr}\left(g^{k}-g^{-k}\right), \quad \forall k=1, \ldots, n .
$$

Taking any initial value $(g(0), J(0), \xi(0))$, the flow of the Hamiltonian $\mathcal{H}_{k}$ can be written as

$$
(g(t), J(t), \xi(t))=\left(g(0) \exp \left(\mathrm{i} t(-\mathrm{i} J(0))^{k-1}\right), J(0), \xi(0)\right) .
$$

For any positive integer $k$, the flow of the Hamiltonian $\hat{\mathcal{H}}_{k}$ reads

$$
(g(t), J(t), \xi(t))=\left(g(0), J(0)+t\left(g(0)^{k}-g(0)^{-k}\right), \xi(0)\right),
$$


and the flow of $\hat{\mathcal{H}}_{-k}$ is

$$
(g(t), J(t), \xi(t))=\left(g(0), J(0)-\mathrm{i} t\left(g(0)^{k}+g(0)^{-k}\right), \xi(0)\right) .
$$

One has the Poisson brackets

$$
\left\{\mathcal{H}_{k}, \mathcal{H}_{l}\right\}_{M}=0, \quad\left\{\hat{\mathcal{H}}_{a}, \hat{\mathcal{H}}_{b}\right\}_{M}=0
$$

for all possible integer subscripts that may occur. In conclusion, the 'spectral invariants' of $J$ and those of $g$ form Abelian algebras of explicitly integrable Hamiltonians.

The 'free' Hamiltonians are invariant under the effective action of $\bar{G}$ on $M$ defined by assigning to each $[y] \in \bar{G}$ the symplectomorphism $A_{[y]}$ of $M$ that operates according td 2

$$
A_{[y]}(g, J, \xi):=\left(y g y^{-1}, y J y^{-1}, y \xi y^{-1}\right),
$$

where $y \in G$ is an arbitrary representative of $[y] \in \bar{G} \simeq G / \mathbb{Z}_{G}$. This action is generated by the equivariant moment map $\Phi: M \rightarrow \overline{\mathcal{G}}^{*} \simeq s u(n)$,

$$
\Phi(g, J, \xi)=J-g^{-1} J g+\xi .
$$

Indeed, $G$ acts by the homomorphism $G \rightarrow \bar{G}$ and the vector field on $M$ corresponding to $T_{a} \in \mathcal{G}$ is the Hamiltonian vector field of $\Phi_{a}:=\left\langle\Phi, T_{a}\right\rangle$, as follows from (2.14) and (2.7). We are going to reduce at the value $\Phi=0$, which is a variant of the KKS reduction [12].

It is known (and is easy to confirm along the lines indicated at the end of Subsection 3.1) that zero is a regular value of the moment map $\Phi$, and $\bar{G}$ acts freely on the constraint-manifold

$$
M_{0}:=\Phi^{-1}(0) \subset M .
$$

Therefore $M_{0}$ is an embedded submanifold of $M$ and the space of orbits

$$
\left(T^{*} G\right)_{\mathrm{red}}:=M_{0} / \bar{G}
$$

is a smooth manifold. This is the reduced phase space of the symplectic reduction of our interest. The same reduced phase space can be obtained directly from $T^{*} G$ by 'point reduction' as well [22], but we shall find it convenient to proceed by utilizing the orbit $\mathcal{O}$ as described above.

The reduction gives rise to the symplectic form $\Omega_{\text {red }}$ and the Abelian algebras of integrable reduced Hamiltonians $\left\{H_{k}\right\}$ and $\left\{\hat{H}_{ \pm k}\right\}$ on $\left(T^{*} G\right)_{\text {red }}$ characterized by the equalities

$$
\pi_{0}^{*}\left(\Omega_{\text {red }}\right)=\iota_{0}^{*}\left(\Omega_{M}\right), \quad H_{k} \circ \pi_{0}=\mathcal{H}_{k} \circ \iota_{0}, \quad \hat{H}_{ \pm k} \circ \pi_{0}=\hat{\mathcal{H}}_{ \pm k} \circ \iota_{0},
$$

where $\pi_{0}: M_{0} \rightarrow M_{0} / \bar{G}$ is the natural submersion and $\iota_{0}: M_{0} \rightarrow M$ is the tautological embedding. The relations in (2.13) imply that

$$
\left\{H_{k}, H_{l}\right\}_{\text {red }}=0, \quad\left\{\hat{H}_{a}, \hat{H}_{b}\right\}_{\text {red }}=0,
$$

where the reduced Poisson bracket is associated with $\Omega_{\text {red }}$. The flows of the reduced Hamiltonians $H_{k}$ and $\hat{H}_{ \pm k}$ result as the $\pi_{0}$-projections of the flows of $\mathcal{H}_{k}$ and $\hat{\mathcal{H}}_{ \pm k}$.

\footnotetext{
${ }^{2}$ The more correct notation $A_{[y]}((g, J, \xi))$ is simplified to $A_{[y]}(g, J, \xi)$ throughout the paper.
} 
In symplectic reduction one often wishes to construct concrete models of the reduced phase space. In principle, any symplectomorphic image of the reduced phase space can serve as a model of it and of course any two models are automatically symplectomorphic to each other. Speaking in terms of our specific example, a global cross-section (also called global gauge slice) is by definition a submanifold $N \subset M_{0}$ that intersects every $\bar{G}$-orbit precisely once and is diffeomorphic to $M_{0} / \bar{G}$ by means of the restriction of $\pi_{0}$. A diffeomorphism between a submanifold $N \subset M_{0}$ intersecting every $\bar{G}$-orbit precisely once and $M_{0} / \bar{G}$ is defined by the restriction of $\pi_{0}$ if and only if the pull-back of $\Omega_{M}$ to $N$ is symplectic. Then $N$ equipped with the pull-back of $\Omega_{M}$ is a model of the reduced phase space $\left(\left(T^{*} G\right)_{\text {red }}, \Omega_{\text {red }}\right)$. In the subsequent Sections 3.1 and 3.2 we shall construct two models of our reduced phase space, but only the second one will be obtained directly as a global gauge slice.

\section{Systems in duality from the reduction of $T^{*} U(n)$}

In this section we present two models of the reduced phase space $\left(\left(T^{*} G\right)_{\text {red }}, \Omega_{\text {red }}\right)$. The first model will be identified with the Sutherland phase space $(P, \omega)(3.8)$ since in terms of this model the reduced Hamiltonians $\left\{H_{k}\right\}(2.18)$ become the spectral invariants of the Sutherland Lax matrix (3.9). The second model $\left(\hat{P}_{c}, \hat{\omega}_{c}\right)$ (3.67) equipped with the commuting Hamiltonians $\left\{\hat{H}_{ \pm k}\right\}$ (2.18) defines the Ruijsenaars dual of the Sutherland system. It yields a completion of the rational Ruijsenaars-Schneider system $\left(\hat{P}, \hat{\omega}, \hat{H}_{\mathrm{RS}}\right)$ described in the Introduction. The identification of $\left(\left(T^{*} G\right)_{\text {red }}, \Omega_{\text {red }}\right)$ with the Sutherland phase space $(P, \omega)$ is well-known [12]. The identification of $\left(\left(T^{*} G\right)_{\text {red }}, \Omega_{\text {red }}\right)$ with $\left(\hat{P}_{c}, \hat{\omega}_{c}\right)$ is constructed by merging the methods applied in the previous papers [20, 21]. In essence, the two models represent two coordinate systems on $\left(T^{*} G\right)_{\text {red }}$, and the change of coordinates gives the duality map (action-angle transform) of Ruijsenaars [8] as we explain at the end of the section.

\subsection{The KKS derivation of the Sutherland system}

Let $\mathbb{T}(n)^{0}$ denote the regular part of the maximal torus

$$
\mathbb{T}(n)=\underbrace{U(1) \times U(1) \times \cdots \times U(1)}_{n \text {-times }}<U(n) .
$$

The open submanifold $\mathbb{T}(n)^{0} \subset \mathbb{T}(n)$, realized as

$$
\mathbb{T}(n)^{0}=\left\{\tau=\operatorname{diag}\left(\tau_{1}, \ldots, \tau_{n}\right) \in \mathbb{T}(n) \mid \tau_{a} \neq \tau_{b} \quad \text { for all } \quad 1 \leq a \neq b \leq n\right\},
$$

is the configuration space of $n$ distinguished 'non-coinciding point-particles' moving on the circle $U(1)$. The permutation group $S(n)$ acts freely on $\mathbb{T}(n)^{0}$, by permuting the entries of $\tau$, and therefore the space of orbits

$$
Q(n):=\mathbb{T}(n)^{0} / S(n)
$$

is a smooth manifold, such that the natural projection $\mathbb{T}(n)^{0} \rightarrow Q(n)$ is a smooth submersion. We note (see Appendix A) that $\mathbb{T}(n)^{0}$ has $(n-1)$ ! connected components and $Q(n)$ is connected. By definition, $Q(n)$ is the configuration space of $n$ indistinguishable particles moving on the circle. 
The cotangent bundle $\left(T^{*} \mathbb{T}(n)^{0}, \Omega_{T^{*} \mathbb{T}(n)^{0}}\right)$ can be identified as

$$
T^{*} \mathbb{T}(n)^{0}=\mathbb{T}(n)^{0} \times \mathbb{R}^{n}=\left\{(\tau, p), \mid \tau \in \mathbb{T}(n)^{0}, p \in \mathbb{R}^{n}\right\}, \quad \Omega_{T^{*} \mathbb{T}(n)^{0}}=\sum_{k=1}^{n} d p_{k} \wedge \frac{d \tau_{k}}{\mathrm{i} \tau_{k}}
$$

If we use the parametrization $\tau_{k}=e^{\mathrm{i} q_{k}}$ with local coordinates $q_{k}$, then the symplectic form takes the usual Darboux form

$$
\Omega_{T^{*} \mathbb{T}(n)^{0}}=\sum_{k=1}^{n} d p_{k} \wedge d q_{k}
$$

The permutation group $S(n)$ acts freely also on $T^{*} \mathbb{T}(n)^{0}$ by the cotangent lift of its action on $\mathbb{T}(n)^{0}$, and it follows from well-known general results 22 that the corresponding space of $S(n)$-orbits is the cotangent bundle of $Q(n)$ :

$$
\left(T^{*} Q(n), \Omega_{T^{*} Q(n)}\right)=\left(T^{*} \mathbb{T}(n)^{0}, \Omega_{T^{*} \mathbb{T}(n)^{0}}\right) / S(n) .
$$

The projection $\mathbb{T}(n)^{0} \rightarrow Q(n)$ is locally a diffeomorphism (a covering) and it induces the map (another covering) $T^{*} \mathbb{T}(n)^{0} \rightarrow T^{*} Q(n)$, whereby the pull-back of $\Omega_{T^{*} Q(n)}$ equals $\Omega_{T^{*} \mathbb{T}(n)^{0}}$. In the coordinates $(\tau, p)$ on $T^{*} \mathbb{T}(n)^{0}(\underline{3.4})$ the cotangent lift of $\sigma \in S(n)$ acts simply as

$$
\sigma:(\tau, p) \mapsto(\sigma(\tau), \sigma(p)),
$$

where $\sigma(\tau)$ and $\sigma(p)$ are obtained by applying the permutation $\sigma$ to the entries of $\tau$ and $p$. In short, one may regard $T^{*} Q(n)$ as $T^{*} \mathbb{T}(n)^{0}$ where the components of $\tau$ and $p$ matter only up to simultaneous permutations. As we explain in Appendix A, $Q(n)$ has a non-trivial topological structure. Just because of that non-trivial structure, it is often advantageous to replace $Q(n)$ by $\mathbb{T}(n)^{0}$ with the proviso that 'everything matters up to permutations'. In particular, we may identify the smooth functions on $T^{*} Q(n)$ with the smooth $S(n)$-invariant functions on $T^{*} \mathbb{T}(n)^{0}$.

Definition 3.1. The Sutherland system as a system of indistinguishable particles possesses the phase space

$$
(P, \omega):=\left(T^{*} Q(n), \Omega_{T^{*} Q(n)}\right)
$$

equipped with the commuting Hamiltonians given by the spectral invariants of the Lax matrix

$$
L_{\text {Suth }}(q, p):=\sum_{k=1}^{n} p_{k} E_{k k}-\frac{\mathrm{i} x}{2} \sum_{a \neq b} \frac{E_{a b}}{\sin \frac{q_{a}-q_{b}}{2}} .
$$

Here $L_{\text {Suth }}(q, p)$ is viewed as a function on $T^{*} \mathbb{T}(n)^{0}(3.4)$, with $\tau=e^{\mathrm{i} q}$, and its symmetric functions yield functions on $T^{*} Q(n)$ due to their $S(n)$-invariance. Throughout the paper, $E_{a b}$ denotes the $n \times n$ matrix having a single non-zero element, equal to 1 , at the $a b$ position.

The following results about the reduction of $\left(M, \Omega_{M}\right)(2.6)$ are due to Kazhdan, Kostant and Sternberg [12]. We present them together with a proof for the sake of readability.

Theorem 3.2. The image of the smooth, injective map $F: T^{*} \mathbb{T}(n)^{0} \rightarrow M$ defined by

$$
F:(\tau, p) \mapsto(\tau, J(\tau, p), \xi(x, \hat{v})), \quad J(\tau, p):=\mathrm{i} \sum_{k=1}^{n} p_{k} E_{k k}+\mathrm{i} \sum_{a \neq b} \frac{x E_{a b}}{1-\tau_{b} / \tau_{a}}, \quad \hat{v}:=[1,1, \ldots, 1]^{T}
$$


is an embedded submanifold $M^{F} \subset M_{0}$ (2.16) that intersects every gauge orbit. If a gauge transformation by $[y] \in \bar{G}$ maps a point of $M^{F}$ into $M^{F}$, then its representative $y \in G$ can be taken to be a permutation matrix. Every permutation matrix $\sigma \in G$ maps $M^{F}$ to $M^{F}$, and $F$ is an $S(n)$-equivariant map with respect to the actions of $S(n)$ on $T^{*} \mathbb{T}(n)^{0}$ and on $M^{F}$. Finally, there holds the relation

$$
F^{*}\left(\Omega_{M}\right)=\Omega_{T^{*} \mathbb{T}(n)^{0}} .
$$

Corollary 3.3. The cotangent bundle $\left(T^{*} Q(n), \Omega_{T^{*} Q(n)}\right)$ is a model of the reduced phase space $\left(\left(T^{*} G\right)_{\mathrm{red}}, \Omega_{\mathrm{red}}\right)$ 2.17). If $(\tau, p)$ is a representative of $[(\tau, p)] \in T^{*} Q(n)=T^{*} \mathbb{T}(n)^{0} / S(n)$, and $\pi_{0}: M_{0} \rightarrow\left(T^{*} G\right)_{\text {red }}$ is the projection, then a symplectomorphism $\mathcal{F}: T^{*} Q(n) \rightarrow\left(T^{*} G\right)_{\text {red }}$ is given by

$$
\mathcal{F}:[(\tau, p)] \mapsto\left(\pi_{0} \circ F\right)(\tau, p) .
$$

By using this symplectomorphism and the identification of the functions on $T^{*} Q(n)$ with $S(n)$ invariant functions on $T^{*} \mathbb{T}(n)^{0}$, the reduced Hamiltonians $H_{k}$ (2.18) take the form

$$
H_{k}(\tau, p)=\frac{1}{k} \operatorname{tr}(-\mathrm{i} J(\tau, p))^{k}, \quad k=1, \ldots, n,
$$

and the reduced Hamiltonians $\left\{\hat{H}_{ \pm k}\right\}$ (2.18) are furnished by

$$
\hat{H}_{k}(\tau, p)=\frac{1}{k} \sum_{j=1}^{n}\left(\left(\tau_{j}\right)^{k}+\left(\tau_{j}\right)^{-k}\right), \quad \hat{H}_{-k}(\tau, p)=\frac{1}{k \mathrm{i}} \sum_{j=1}^{n}\left(\left(\tau_{j}\right)^{k}-\left(\tau_{j}\right)^{-k}\right) .
$$

By setting $\tau_{j}=e^{\mathrm{i} q_{j}}$, the functions $H_{k}$ become spectral invariants of the Lax matrix $L_{\mathrm{Suth}}(q, p)$.

Proof. We have to check the validity of the middle two in the following chain of identifications:

$$
\left(T^{*} G\right)_{\mathrm{red}}=M_{0} / \bar{G} \simeq M^{F} / S(n) \simeq T^{*} \mathbb{T}(n)^{0} / S(n)=T^{*} Q(n) .
$$

We start by recalling that the moment map constraint is

$$
J-g^{-1} J g+\xi(x, v)=0 \quad \text { with } \quad \xi(x, v)=\mathrm{i} x\left(\mathbf{1}_{n}-v v^{\dagger}\right), \quad|v|^{2}=n .
$$

By using the gauge freedom, we can transform any solution of this constraint into a solution for which $g$ belongs to the maximal torus. That is, it is sufficient to solve the constraint

$$
J-\tau^{-1} J \tau=\mathrm{i} x\left(v v^{\dagger}-\mathbf{1}_{n}\right), \quad|v|^{2}=n, \quad \tau \in \mathbb{T}(n) .
$$

We can see from the diagonal part of this equation that the diagonal components of $J$ are arbitrary, and also see that $\left|v_{k}\right|=1$ for each $k=1, \ldots, n$. Therefore we can bring the solution, by a gauge transformation by an element of $\mathbb{T}(n)$, into a solution for which $v=\hat{v}$ defined in (3.10). Then the off-diagonal components of the constraint become

$$
J_{a b}\left(1-\tau_{b} / \tau_{a}\right)=\mathrm{i} x, \quad \forall a \neq b .
$$

This can be solved if and only of $\tau \in \mathbb{T}(n)^{0}(3.2)$, and the solution is given precisely by the formula $J(\tau, p)$ in (3.10). To summarize, so far we have shown that

$$
(g, J, \xi(x, v))=(\tau, J(\tau, p), \xi(x, \hat{v}))
$$


is a solution of the moment map constraint for all $\tau \in \mathbb{T}(n)^{0}$ and $p \in \mathbb{R}^{n}$, and every solution is a gauge transform of a solution of this form. Notice that these solutions form precisely the image $M^{F}$ of the map $F(\underline{3.10})$. Note also that

$$
L_{\text {Suth }}(q, p)=-\mathrm{i} e^{-\mathrm{i} q / 2} J\left(e^{\mathrm{i} q}, p\right) e^{\mathrm{i} q / 2} .
$$

Consider now a 'residual gauge transformation' $[y] \in \bar{G}$ that maps a solution of the above form into another (or the same) solution of the above form, i.e.,

$$
\left(y \tau y^{-1}, y J(\tau, p) y^{-1}, \xi(x, y \hat{v})\right)=\left(\tau^{\prime}, J\left(\tau^{\prime}, p^{\prime}\right), \xi(x, \hat{v})\right) .
$$

We conclude from the equality $y \tau y^{-1}=\tau^{\prime}$ that $y$ must have the form $y=\sigma T$, where $\sigma \in G$ is a permutation matrix and $T \in \mathbb{T}(n)$. Then we infer from the third component of (3.21) that $T$ must be a multiple of the unit matrix. Returning to the first and second components, we see that

$$
\tau^{\prime}=\sigma \tau \sigma^{-1} \equiv \sigma(\tau) \quad \text { and } \quad \sum_{k} p_{k}^{\prime} E_{k k}=\sigma\left(\sum_{k} p_{k} E_{k k}\right) \sigma^{-1} \equiv \sum_{k=1}^{n} \sigma(p)_{k} E_{k k}
$$

This shows that $F$ is an $S(n)$-equivariant bijection between $T^{*} \mathbb{T}(n)^{0}$ and $M^{F}$, and it is also easily checked that $F^{*}\left(\Omega_{M}\right)=\Omega_{T^{*} \mathbb{T}(n)^{0}}$ holds. Now all statements of the theorem and the corollary follow immediately from the properties of $F$ and its image $M^{F}$ that we have established, and from (3.20). Incidentally, it is also clear from the above that the isotropy subgroup of any $(\tau, J(\tau, p), \xi(x, \hat{v})) \in M^{F}$ is the trivial subgroup of $\bar{G}$, which entails that $\bar{G}$ acts freely on $M_{0}$.

Q.E.D.

For completeness, the reader may wish to verify that zero is a regular value of the moment map $\Phi$, i.e., the derivative map $D \Phi(g, J, \xi): T_{(g, J, \xi)} M \rightarrow s u(n)$ is surjective at every point of $M_{0}=\Phi^{-1}(0)$. The required inspection is readily performed at the points of $M^{F} \subset M_{0}$, which is sufficient since $\Phi$ is equivariant and $M^{F}$ intersects every gauge orbit in $M_{0}$.

\subsection{Derivation of the Ruijsenaars dual of the Sutherland system}

We have seen that the Sutherland phase space $(P, \omega)(3.8)$ is a model of $\left(T^{*} G\right)_{\text {red }}$ defined by the KKS reduction of $\left(M, \Omega_{M}\right)$. Now our aim is to construct a 'dual' model of the reduced phase space $\left(T^{*} G\right)_{\text {red }}$. For this purpose we devise an alternative way to solve the moment map constraint (3.16). In the preceding subsection we proceeded by diagonalizing $g \in G$, and here we start from the observation that every solution of (3.16) can be obtained as a gauge transform of a solution for which $J \in \mathcal{G}$ is diagonal of the form

$$
J=-\mathrm{i} \operatorname{diag}\left(\hat{p}_{1}, \ldots, \hat{p}_{n}\right):=-\mathrm{i} \hat{p} \quad \text { with } \quad \hat{p}_{1} \geq \hat{p}_{2} \geq \cdots \geq \hat{p}_{n} .
$$

The final result, given by Theorem 3.12, will be reached through a series of auxiliary lemmas.

Lemma 3.4. If $(g, J, \xi(x, v))$ is a solution of the moment moment map constraint (3.16) with $J$ in (3.23) then

$$
\hat{p}_{k}-\hat{p}_{k+1} \geq|x|, \quad \forall k=1, \ldots,(n-1),
$$


and

$$
\left|v_{b}\right|^{2}=\prod_{k \neq b}\left[\frac{\hat{p}_{b}-\hat{p}_{k}-x}{\hat{p}_{b}-\hat{p}_{k}}\right], \quad \forall b=1, \ldots, n
$$

Proof. We rewrite the moment map constraint $(3.16)$ in the equivalent form

$$
g^{-1} \hat{p} g=x\left(v v^{\dagger}-\mathbf{1}_{n}\right)+\hat{p} .
$$

We can compute the characteristic polynomials of the matrices on the two sides of this equation. In this way we deduce the equality of the polynomials

$$
\prod_{j=1}^{n}\left(\hat{p}_{j}-\lambda\right)=\prod_{j=1}^{n}\left(\hat{p}_{j}-(\lambda+x)\right)+x \sum_{k=1}^{n}\left(\left|v_{k}\right|^{2} \prod_{j \neq k}\left(\hat{p}_{j}-(\lambda+x)\right)\right) .
$$

Suppose now that $\hat{p}$ is regular, i.e.,

$$
\hat{p}_{1}>\hat{p}_{2}>\cdots>\hat{p}_{n}
$$

For regular $\hat{p}(3.28)$, the evaluation of (3.27) at $\lambda=\hat{p}_{b}-x$ gives immediately the relation (3.25). By using (3.25) together with (3.28) and $\left|v_{b}\right|^{2} \geq 0$, it is not difficult to obtain the 'spectral gap condition' (3.24). Indeed, one may follow the argumentation presented in [21] in connection with a completely analogous problem.

Let us continue by showing that solutions of (3.26) satisfying (3.28) exist. In fact, one may take the explicit example defined for any $\hat{p}$ with

$$
\hat{p}_{k}-\hat{p}_{k+1}=|x|, \quad k=1, \ldots, n-1,
$$

as follows:

$$
v_{i}=\delta_{n, i} \sqrt{n}, \quad g_{1, n}=g_{j, j-1}=1 \quad(j=2, \ldots, n), \quad g_{a, b}=0 \quad \text { otherwise, } \quad \text { if } \quad x>0,
$$

and

$$
v_{i}=\delta_{1, i} \sqrt{n}, \quad g_{n, 1}=g_{j, j+1}=1 \quad(j=1, \ldots, n-1), \quad g_{a, b}=0 \quad \text { otherwise }, \quad \text { if } \quad x<0 .
$$

We observe from the foregoing arguments that for all solutions of (3.16) for which the eigenvalues of $J$ are all distinct the distance of any two eigenvalues of $J$ is at least $|x|$, and such regular solutions do exist. This allows us to conclude that there cannot be any solution for which two eigenvalues of $J$ coincide. Indeed, we know that the constraint-manifold $M_{0}$ is connected. (Recall from Section 3.1 that $M_{0}$ is a principal fiber bundle with connected fiber $\bar{G}$ and connected base $T^{*} Q(n)$.) Therefore any hypothetical non-regular solution would be continuously connected to a regular solution. However, this contradicts the lower bound $|x|$ in the distance of the eigenvalues of any regular solution.

Q.E.D.

Definition 3.5. Let $\overline{\mathfrak{C}}_{x}$ denote the closure of the 'Weyl chamber with thick walls' $\mathfrak{C}_{x}$ introduced in (1.6). Define the function $V(x): \overline{\mathfrak{C}}_{x} \rightarrow \mathbb{R}^{n}$ by the formula

$$
V(x, \hat{p})_{b}:=\prod_{k \neq b}\left[\frac{\hat{p}_{b}-\hat{p}_{k}-x}{\hat{p}_{b}-\hat{p}_{k}}\right]^{\frac{1}{2}}, \quad \forall b=1, \ldots, n,
$$


and introduce the real $n \times n$ matrix valued smooth function $\eta(x, \hat{p})$ on $\overline{\mathfrak{C}}_{x}$ by the formula

$$
\begin{gathered}
\eta(x, \hat{p})_{a b}=\frac{x}{\hat{p}_{b}-\hat{p}_{a}} \prod_{j \neq a, b}\left[\frac{\left(\hat{p}_{a}-\hat{p}_{j}-x\right)\left(\hat{p}_{j}-\hat{p}_{b}-x\right)}{\left(\hat{p}_{a}-\hat{p}_{j}\right)\left(\hat{p}_{j}-\hat{p}_{b}\right)}\right]^{\frac{1}{2}}, \quad a \neq b, \\
\eta(x, \hat{p})_{a a}=\prod_{j \neq a}\left[\frac{\left(\hat{p}_{a}-\hat{p}_{j}-x\right)\left(\hat{p}_{j}-\hat{p}_{a}-x\right)}{\left(\hat{p}_{a}-\hat{p}_{j}\right)\left(\hat{p}_{j}-\hat{p}_{a}\right)}\right]^{\frac{1}{2}} .
\end{gathered}
$$

Note that the expression under the square root is non-negative in each factor in the above three equations and the non-negative square root is taken.

Lemma 3.6. There exists a solution of the moment map constraint (3.16) of the form

$$
(g, J, \xi)=(g,-\mathrm{i} \hat{p}, \xi(x, V(x, \hat{p})))
$$

for every $\hat{p} \in \overline{\mathfrak{C}}_{x}$. Here, we use the notation $\hat{p}=\operatorname{diag}\left(\hat{p}_{1}, \ldots, \hat{p}_{n}\right)$.

Proof. Let us arbitrarily fix $\hat{p} \in \overline{\mathfrak{C}}_{x}$. Recall that the moment map constraint requires the existence of $g \in U(n)$ for which

$$
g^{-1} \hat{p} g=x\left(V(x, \hat{p}) V(x, \hat{p})^{\dagger}-\mathbf{1}_{n}\right)+\hat{p} .
$$

Since the matrix on the right-hand-side is Hermitian, the existence of such a $g$ is guaranteed if we show that the characteristic polynomial $\mathcal{Q}_{n}(\lambda, \hat{p})$ of the matrix on right hand side is equal to $\mathcal{P}_{n}(\lambda):=\prod_{j=1}^{n}\left(\hat{p}_{j}-\lambda\right)$. The very definition of $V(x, \hat{p})$ (see the argument after (3.28) ) guarantees that

$$
\left(\mathcal{P}_{n}-\mathcal{Q}_{n}\right)\left(\lambda=\hat{p}_{b}-x, \hat{p}\right)=0, \quad \forall b=1, \ldots, n .
$$

On the other hand, it is obvious from their definition that $\mathcal{P}_{n}-\mathcal{Q}_{n}$ is a polynomial in $\lambda$ of degree strictly lower than $n$. Therefore we have the equality $\mathcal{P}_{n}(\lambda, \hat{p})=\mathcal{Q}_{n}(\lambda, \hat{p})$. Q.E.D.

Lemma 3.7. The function $V(x, \hat{p})$ (3.32) satisfies the identities

$$
\begin{gathered}
\sum_{a=1}^{n} \frac{x}{\hat{p}_{b}-\hat{p}_{a}+x} V(x, \hat{p})_{a}^{2}=1, \quad \forall b=1, \ldots, n, \quad \forall \hat{p} \in \mathfrak{C}_{x}, \\
\sum_{a=1}^{n} V(x, \hat{p})_{a}^{2}=n, \quad \forall \hat{p} \in \overline{\mathfrak{C}}_{x} .
\end{gathered}
$$

The function $\eta(x, \hat{p})$ enjoys the properties

$$
\begin{gathered}
\eta(x, \hat{p})_{a b}=\frac{x V(x, \hat{p})_{a} V(-x, \hat{p})_{b}}{\hat{p}_{b}-\hat{p}_{a}+x}, \quad \forall a, b \quad \text { if } \quad \hat{p} \in \mathfrak{C}_{x}, \\
\eta(x, \hat{p})^{-1}=\eta(x, \hat{p})^{T}=\eta(-x, \hat{p}), \quad \operatorname{det}(\eta(x, \hat{p}))=1, \quad \forall \hat{p} \in \overline{\mathfrak{C}}_{x} .
\end{gathered}
$$

Proof. The identity (3.39) follows by taking the trace of (3.26), and (3.38) follows by evaluation of the equality of the characteristic polynomials (3.27) at $\lambda=\lambda_{b}$. We note in passing that the identity (3.38) also extends smoothly to the closure $\overline{\mathfrak{C}}_{x}$ since the singularities coming from the 
denominators cancel against the zeros of the components of $V$. Regarding $\eta(x, \hat{p})$, the only nontrivial statements are the first equality in (3.41) and the claim about the determinant being 1. These statements follow from (3.40) by using the Cauchy determinant formula (see e.g. [23]) and the continuity of $\eta$ on $\overline{\mathfrak{C}}_{x}$.

Q.E.D.

Note that the functions $V(x, \hat{p})$ and $\eta(x, \hat{p})$ as well as their properties given by Lemma 3.7 can be found in [8], too. Observe from (3.41) that $\eta(x, \hat{p}) \in S O(n, \mathbb{R})<S U(n)$.

Lemma 3.8. By using the above notations, the following formula defines a solution of the moment map constraint (3.16) for each $\hat{p} \in \overline{\mathfrak{C}}_{x}$ :

$$
(g, J, \xi)=\left(\eta(x, \hat{p})^{-1},-\mathrm{i} \hat{p}, \xi(x, V(x, \hat{p}))\right) .
$$

Proof. By multiplying (3.26) by $g^{-1}$ from the right and substituting (3.42), we see that the statement is equivalent to the identity

$$
\eta(x, \hat{p})_{a b}\left(\hat{p}_{b}-\hat{p}_{a}+x\right)=x V_{a}(x, \hat{p}) \sum_{c=1}^{n} V_{c}(x, \hat{p}) \eta(x, \hat{p})_{c b}, \quad \forall a, b .
$$

By using the above formulae, it is readily verified that both sides are equal to

$$
x V(x, \hat{p})_{a} V(-x, \hat{p})_{b}
$$

The easiest way is to first check this identity on $\mathfrak{C}_{x}$, and then notice that continuity guarantees that it remains valid also on the closure $\overline{\mathfrak{C}}_{x}$.

Lemma 3.9. Applying the notations of (2.14) and Definition 3.5, define the continuous map

$$
K_{x}: \bar{G} \times \mathbb{T}(n) \times \overline{\mathfrak{C}}_{x} \rightarrow M \simeq U(n) \times u(n) \times \mathcal{O}
$$

by the formula

$$
K_{x}([y], \mathcal{D}, \hat{p})=A_{[y]}\left((\eta(x, \hat{p}) \mathcal{D})^{-1},-\mathrm{i} \hat{p}, \xi(x, V(x, \hat{p}))\right) .
$$

Then the image of $K_{x}$ coincides with the constraint-manifold $M_{0}$ (2.16). The restriction of $K_{x}$ to $\bar{G} \times \mathbb{T}(n) \times \mathfrak{C}_{x}$ is smooth, injective and its image is a dense, open submanifold of $M_{0}$.

Proof. It follows from Lemmas 3.4 and 3.6 that every element of $M_{0}$ can be obtained as a gauge transform of an element of $M_{0}$ of the form

$$
(g,-\mathrm{i} \hat{p}, \xi(x, V(x, \hat{p}))) \quad \text { with some } \hat{p} \in \overline{\mathfrak{C}}_{x} .
$$

In this case the moment map constraint amounts to the following equation for $g \in U(n)$ :

$$
\mathrm{i} g^{-1} \hat{p} g=-\xi(x, V(x, \hat{p}))+\mathrm{i} \hat{p} .
$$

By noting that $\hat{p}$ is regular and that we have Lemma 3.8, we see that (3.48) is solved if and only if

$$
g=(\eta(x, \hat{p}) \mathcal{D})^{-1} \quad \text { for some } \quad \mathcal{D} \in \mathbb{T}(n) .
$$


These arguments show that the image of $K_{x}$ is indeed $M_{0}$. It is also obvious that $K_{x}$ maps $\bar{G} \times \mathbb{T}(n) \times \mathfrak{C}_{x}$ onto a dense, open submanifold of $M_{0}$. The restricted map is smooth on account of its formula, and to show that it is injective suppose that

$$
A_{[y]}\left((\eta(x, \hat{p}) \mathcal{D})^{-1},-\mathrm{i} \hat{p}, \xi(x, V(x, \hat{p}))\right)=A_{[w]}\left(\left(\eta\left(x, \hat{p}^{\prime}\right) \mathcal{D}^{\prime}\right)^{-1},-\mathrm{i} \hat{p}^{\prime}, \xi\left(x, V\left(x, \hat{p}^{\prime}\right)\right)\right)
$$

for some

$$
([y], \mathcal{D}, \hat{p}) \in \bar{G} \times \mathbb{T}(n) \times \mathfrak{C}_{x} \ni\left([w], \mathcal{D}^{\prime}, \hat{p}^{\prime}\right) .
$$

Comparison of the second component of the triples in (3.50) implies that $\hat{p}=\hat{p}^{\prime}$ and

$$
g_{0}:=w^{-1} y \in \mathbb{T}(n) .
$$

Moreover, it then follows from the third component of (3.50) that

$$
g_{0} \xi(x, V(x, \hat{p})) g_{0}^{-1}=\xi\left(x, g_{0} V(x, \hat{p})\right)=\xi(x, V(x, \hat{p})) .
$$

This means that

$$
g_{0} V(x, \hat{p})=\gamma V(x, \hat{p}) \quad \text { for some } \quad \gamma \in U(1) .
$$

Taking into account that all components of $V(x, \hat{p})$ are non-zero, since $\hat{p} \in \mathfrak{C}_{x}$, we get that $g_{0}=\gamma \mathbf{1}_{n}$. The statement now follows by looking at the first component of the equality (3.50).

Q.E.D.

Define

$$
M_{0}^{0} \subset M_{0}
$$

to be the set of the elements $(g, J, \xi) \in M_{0}$ for which the eigenvalues of $J$ satisfy the strict spectral gap condition, i.e., for which $\mathrm{i} J$ is conjugate to an element of $\mathfrak{C}_{x}$. It is clear that $M_{0}^{0} \subset M_{0}$ is a dense, open, $\bar{G}$-stable submanifold, which is the image of $\bar{G} \times \mathbb{T}(n) \times \mathfrak{C}_{x}$ by the map $K_{x}$. Correspondingly,

$$
M_{0}^{0} / \bar{G} \subset M_{0} / \bar{G}
$$

is a dense, open submanifold of the reduced phase space.

Lemma 3.10. Consider the smooth, injective map $m_{x}: \mathbb{T}(n) \times \mathfrak{C}_{x} \rightarrow M$ given by

$$
m_{x}(\mathcal{D}, \hat{p}):=K_{x}([e], \mathcal{D}, \hat{p})=\left((\eta(x, \hat{p}) \mathcal{D})^{-1},-\mathrm{i} \hat{p}, \xi(x, V(x, \hat{p}))\right) .
$$

The image of $m_{x}$ lies in $M_{0}^{0}$ and it intersects every gauge orbit in $M_{0}^{0}$ precisely once. Moreover, $m_{x}$ pulls-back the symplectic form $\Omega_{M}$ of $M$ (2.6) according to

$$
m_{x}^{*}\left(\Omega_{M}\right)=-\mathrm{i} \operatorname{tr}\left(d \hat{p} \wedge(d \mathcal{D}) \mathcal{D}^{-1}\right)
$$

Proof. The only task is to verify (3.58). Indeed, the smoothness of $m_{x}$ is obvious from its formula and it enjoys the properties mentioned above as an immediate consequence of Lemma 3.9. To verify $(\underline{3.58})$ we put $g:=(\eta(x, \hat{p}) \mathcal{D})^{-1}$ and $J=-\mathrm{i} \hat{p}$, which satisfy the equality

$$
\operatorname{tr}\left(J(d g) g^{-1}\right)=\operatorname{tr}\left(\mathrm{i} \hat{p}(d \mathcal{D}) \mathcal{D}^{-1}\right) .
$$

The basic fact that implies this is that $\eta$ is a real orthogonal matrix, and thus it gives no cross term with $\hat{p}$. It is also important to notice that the 'orbital part' $\Omega_{\mathcal{O}}$ of the symplectic form $\Omega_{M}$ 
gives zero contribution to $m_{x}^{*}\left(\Omega_{M}\right)$. This follows from the formula (2.5) of the symplectic form $\Omega_{\mathcal{O}}$ in terms of the redundant variable $v$, as it becomes zero upon restriction to any submanifold consisting of vectors $v \in \mathbb{C}^{n}$ with purely real components. Since the components of $V(x, \hat{p})$ are all real, the statement (3.58) follows.

Q.E.D.

For each $\tau=\operatorname{diag}\left(\tau_{1}, \ldots, \tau_{n}\right) \in \mathbb{T}(n)$ set

$$
\tau_{(x)}:=\operatorname{diag}\left(\tau_{2}, \ldots, \tau_{n}, 1\right) \quad \text { if } \quad x>0, \quad \tau_{(x)}:=\operatorname{diag}\left(1, \tau_{1}, \ldots, \tau_{n-1}\right) \quad \text { if } \quad x<0 .
$$

Introduce also the bijection $\aleph(x, \cdot): \mathbb{T}(n) \rightarrow \mathbb{T}(n)$ by

$$
\aleph(x, \tau)_{j}:=\prod_{k=j}^{n} \tau_{k}^{-1}, \quad x>0 \quad \text { and } \quad \aleph(x, \tau)_{j}:=\prod_{k=1}^{j} \tau_{k}^{-1}, \quad x<0 .
$$

Note the identity

$$
\aleph(x, \tau)(\aleph(x, \tau))_{(x)}^{-1}=\tau^{-1}, \quad \forall \tau \in \mathbb{T}(n) .
$$

We described in the Introduction the Hamiltonian system $\left(\hat{P}, \hat{\omega}, \hat{H}_{\mathrm{RS}}\right)$, which is a real form of the complex rational Ruijsenaars-Schneider system. The commuting Hamiltonians of this system are the spectral invariants of the Lax matrix

$$
\hat{L}(\hat{q}, \hat{p}):=\eta(x, \hat{p}) e^{\mathrm{i} \hat{q}}
$$

viewed as a function on $\hat{P}$ (1.6). In particular, the Hamiltonian (1.7) obeys

$$
\hat{H}_{\mathrm{RS}}(\hat{q}, \hat{p})=\frac{1}{2} \operatorname{tr}\left(\hat{L}(\hat{q}, \hat{p})+\hat{L}(\hat{q}, \hat{p})^{-1}\right) .
$$

The usefulness of the next reformulation of Lemma 3.10 will be justified by the final result.

Proposition 3.11. With $(\hat{P}, \hat{\omega})$ in (1.6) and $K_{x}$ in (3.46), the map $k_{x}: \hat{P} \rightarrow M$ defined by

$$
k_{x}\left(e^{\mathrm{i} \hat{q}}, \hat{p}\right):=K_{x}\left(\left[\aleph\left(x, e^{\mathrm{i} \hat{q}}\right)_{(x)}\right], e^{\mathrm{i} \hat{q}}, \hat{p}\right)
$$

enjoys the same properties as the map $m_{x}$ of Lemma 3.10. In particular, $k_{x}^{*}\left(\Omega_{M}\right)=\hat{\omega}$. Thus the symplectic manifold $(\hat{P}, \hat{\omega})$ provides a model of the dense, open submanifold $M_{0}^{0} / \bar{G}$ of the reduced phase space. On this dense open submanifold the Abelian family $\left\{\hat{\mathcal{H}}_{ \pm k}\right\}$ (2.9) reduces to the spectral invariants of the Lax matrix $\hat{L}(\hat{q}, \hat{p})$ (3.63), and the other Abelian family $\left\{\mathcal{H}_{k}\right\}$ (2.8) reduces to the symmetric polynomials of the 'dual position variable' $\hat{p}$.

Proof. The claims follow from Lemma 3.10 since the maps $m_{x}$ and $k_{x}$ are gauge equivalent.

Q.E.D.

The results presented above characterize the dense submanifold $M_{0}^{0} / \bar{G} \subset M_{0} / \bar{G}$ in 'dual variables'. For a full description, we shall construct a global cross-section of the gauge orbits in the complete constraint-manifold $M_{0}$. This will be achieved by constructing an extension of the map $k_{x}$ (in the sense of Eq. (3.86) below). To save place, in the rest of this section we assume that

$$
x>0 .
$$


We introduce the symplectic manifold $\left(\hat{P}_{c}, \hat{\omega}_{c}\right)$ by setting

$$
\hat{P}_{c}:=\mathbb{C}^{n-1} \times \mathbb{C}^{\times}, \quad \hat{\omega}_{c}:=\frac{\mathrm{i} d Z \wedge d \bar{Z}}{2 \bar{Z} Z}+\sum_{j=1}^{n-1} \mathrm{i} d z_{j} \wedge d \bar{z}_{j}, \quad Z \in \mathbb{C}^{\times}, \quad z \in \mathbb{C}^{n-1},
$$

where $\mathbb{C}^{\times}$denotes the complex plane without the origin. Following [8, 21], we then define the smooth, injective map $\mathcal{Z}_{x}: \hat{P} \equiv \mathbb{T}(n) \times \mathfrak{C}_{x} \rightarrow \hat{P}_{c}$ by the formulae

$$
z_{j}\left(e^{\mathrm{i} \hat{q}}, \hat{p}\right):=\left(\hat{p}_{j}-\hat{p}_{j+1}-x\right)^{\frac{1}{2}} \prod_{k=j+1}^{n} e^{-\mathrm{i} \hat{q}_{k}}, j=1, \ldots, n-1, Z\left(e^{\mathrm{i} \hat{q}}, \hat{p}\right):=e^{-\hat{p}_{1}} \prod_{k=1}^{n} e^{-\mathrm{i} \hat{q}_{k}}
$$

and let $\tilde{\mathcal{Z}}_{x}: \mathbb{T}(n) \times \overline{\mathfrak{C}}_{x} \rightarrow \hat{P}_{c}$ stand for the unique continuous extension of $\mathcal{Z}_{x}$. The componentfunctions of $\tilde{\mathcal{Z}}_{x}$ are given by the same formulae as those of $\mathcal{Z}_{x}$. The map $\tilde{\mathcal{Z}}_{x}$ is surjective, but is obviously not injective. The image

$$
\hat{P}_{c}^{0}:=\mathcal{Z}_{x}(\hat{P}) \subset \hat{P}_{c}
$$

is the dense, open submanifold consisting of the points for which $z_{j} \neq 0$ for all $j$. We have

$$
\mathcal{Z}_{x}^{*}\left(\hat{\omega}_{c}\right)=\sum_{i=1}^{n} d \hat{p}_{i} \wedge d \hat{q}_{i} \equiv \hat{\omega}
$$

This means that $\mathcal{Z}_{x}$ yields a symplectomorphism between $(\hat{P}, \hat{\omega})$ and $\left(\hat{P}_{c}^{0}, \hat{\omega}_{c}\right)$.

Now we introduce the functions $\hat{\pi}_{j}(z, Z)$, the matrix function $\vartheta(z, Z)$, and the vectorfunction $\mathcal{V}(z, Z)$ on $\hat{P}_{c}$ by the following defining formulae:

$$
\begin{aligned}
& \hat{\pi}_{j}\left(z\left(e^{\mathrm{i} \hat{q}}, \hat{p}\right), Z\left(e^{\mathrm{i} \hat{q}}, \hat{p}\right)\right)=\hat{p}_{j}, \\
& \mathcal{V}\left(z\left(e^{\mathrm{i} \hat{q}}, \hat{p}\right), Z\left(e^{\mathrm{i} \hat{q}}, \hat{p}\right)\right)=\aleph\left(x, e^{\mathrm{i} \hat{q}}\right)_{(x)} V(x, \hat{p}), \\
& \vartheta\left(z\left(e^{\mathrm{i} \hat{q}}, \hat{p}\right), Z\left(e^{\mathrm{i} \hat{q}}, \hat{p}\right)\right)=\aleph\left(x, e^{\mathrm{i} \hat{q}}\right)_{(x)}\left(\eta(x, \hat{p}) e^{\mathrm{i} \hat{q}}\right) \aleph\left(x, e^{\mathrm{i} \hat{q}}\right)_{(x)}^{-1},
\end{aligned}
$$

using (3.60) and (3.61). The main point is that that this definition makes sense for all $\left(e^{\mathrm{i} \hat{q}}, \hat{p}\right) \in$ $\mathbb{T}(n) \times \overline{\mathfrak{C}}_{x}$. One can also check by writing explicit formulae that $\hat{\pi}_{j}, \mathcal{V}$ and $\vartheta$ are smooth functions on $\hat{P}_{c}$.

To present the explicit formulae of the above functions, we first of all note that

$$
\hat{\pi}_{1}(z, Z)=-\log |Z|, \quad \hat{\pi}_{k}(z, Z)=-(k-1) x-\log |Z|-\sum_{j=1}^{k-1}\left|z_{j}\right|^{2}, \quad k=2, \ldots, n .
$$

Then we define the auxiliary functions

$$
Q_{j, k}:=\left[\frac{\hat{\pi}_{j}-\hat{\pi}_{k}-x}{\hat{\pi}_{j}-\hat{\pi}_{k}}\right]^{\frac{1}{2}}, \quad \forall j \neq k \in\{1, \ldots, n\} .
$$

As a result of the spectral gap condition (3.24), all expressions under the square root are nonnegative, and their denominators are non-zero. Of course, one can easily spell out the functions 
$Q_{j, k}$ more explicitly. They depend only on the absolute values of the variables $Z, z_{i}$, and satisfy, on the whole of $\hat{P}_{c}$, the inequalities

$$
Q_{j, k}>0 \quad \text { if } \quad k-j \neq 1
$$

Then we have

$$
\mathcal{V}_{j}=\frac{z_{j}}{\sqrt{\left|z_{j}\right|^{2}+x}} \prod_{k \neq j, j+1} Q_{j, k}, \quad j=1, \ldots, n-1 \quad \text { and } \quad \mathcal{V}_{n}=\prod_{k \neq n} Q_{n, k}
$$

The various entries of the matrix $\vartheta(z, Z) \in U(n)$ can be listed as follows:

$$
\begin{gathered}
\vartheta_{i, i+1}=\frac{x}{\hat{\pi}_{i+1}-\hat{\pi}_{i}} \prod_{j \neq i, i+1}\left(Q_{i, j} Q_{j, i+1}\right), \quad i=1, \ldots, n-1, \\
\vartheta_{n, 1}=\frac{x}{\hat{\pi}_{1}-\hat{\pi}_{n}} \frac{\bar{Z}}{|Z|} \prod_{j \neq 1, n}\left(Q_{n, j} Q_{j, 1}\right), \\
\vartheta_{n, k}=\frac{x}{\hat{\pi}_{k}-\hat{\pi}_{n}} \frac{\bar{z}_{k-1} Q_{n, k-1}}{\sqrt{\left|z_{k-1}\right|^{2}+x}} \prod_{j \neq k-1, k, n}\left(Q_{n, j} Q_{j, k}\right), \quad k \in\{1, \ldots, n\} \backslash\{1, n-1, n\}, \\
\vartheta_{k, k}=\frac{\vartheta_{k, 1}=\frac{x}{\hat{\pi}_{1}-\hat{\pi}_{k}} \frac{z_{k} Q_{k+1,1}}{\sqrt{\left|z_{k}\right|^{2}+x}} \frac{\bar{Z}}{|Z|} \prod_{j \neq 1, k, k+1}\left(Q_{k, j} Q_{j, 1}\right), \quad k=2, \ldots, n-1,}{\left.\bar{z}_{k-1}\right|^{2}+x}\left(\prod_{j \neq k, k+1} Q_{k, j}\right)\left(\prod_{l \neq k-1, k} Q_{l, k}\right), \quad k=2, \ldots, n-1, \\
\vartheta_{1,1}=\frac{z_{1} Z}{|Z|} \frac{\left.\left.\sqrt{|Z|}\right|_{k-1}\right|^{2}+x}{\left|z_{1}\right|^{2}+2 x} \\
\prod_{j=3}^{n}\left(Q_{1, j} Q_{j, 1}\right), \quad \vartheta_{n, n}=\bar{z}_{n-1} \frac{\sqrt{\left|z_{n-1}\right|^{2}+2 x}}{\left|z_{n-1}\right|^{2}+x} \prod_{j=1}^{n-2}\left(Q_{n, j} Q_{j, n}\right),
\end{gathered}
$$

and finally

$$
\vartheta_{a, b}=\frac{z_{a}}{\sqrt{\left|z_{a}\right|^{2}+x}} \frac{\bar{z}_{b-1}}{\sqrt{\left|z_{b-1}\right|^{2}+x}} \frac{x Q_{a, b-1} Q_{a+1, b}}{\hat{\pi}_{b}-\hat{\pi}_{a}} \prod_{j \neq a, a+1, b-1, b}\left(Q_{a, j} Q_{j, b}\right)
$$

for the remaining indices $1 \leq a, b \leq n$ subject to $a \neq b, b \neq a+1, a \neq n, b \neq 1$.

Now we are in the position to define the smooth map $\hat{\mathcal{I}}: \hat{P}_{c} \rightarrow M_{0}$ by

$$
\hat{\mathcal{I}}(z, Z):=\left(\vartheta(z, Z)^{-1},-\mathrm{i} \hat{\pi}(z, Z), \xi(x, \mathcal{V}(z, Z))\right) .
$$

The rationale behind this definition, and also for the definition of the various functions above, comes from the following relation:

$$
\hat{\mathcal{I}} \circ \mathcal{Z}_{x}=k_{x}
$$

Thus $\hat{\mathcal{I}}$ is the unique continuous extension of the map $k_{x} \circ \mathcal{Z}_{x}^{-1}: \hat{P}_{c}^{0} \rightarrow M_{0}$ to $\hat{P}_{c}$ (cf. (3.69)). Moreover, the map $\hat{\mathcal{I}}$ enjoys the property

$$
\hat{\mathcal{I}}\left(z\left(e^{\mathrm{i} \hat{q}}, \hat{p}\right), Z\left(e^{\mathrm{i} \hat{q}}, \hat{p}\right)\right)=K_{x}\left(\left[\aleph\left(x, e^{\mathrm{i} \hat{q}}\right)_{(x)}\right], e^{\mathrm{i} \hat{q}}, \hat{p}\right) \quad \forall\left(e^{\mathrm{i} \hat{q}}, \hat{p}\right) \in \mathbb{T}(n) \times \overline{\mathfrak{C}}_{x} .
$$

Recalling (3.65), note that (3.86) is the restriction of (3.87) to the dense, open subset $\mathbb{T}(n) \times \mathfrak{C}_{x}$. 
Theorem 3.12. Consider the symplectic manifold $\left(\hat{P}_{c}, \hat{\omega}_{c}\right)(\sqrt{3.67})$ and the map $\hat{\mathcal{I}}: \hat{P}_{c} \rightarrow M_{0}$ defined by (3.85) using the notations listed in (3.74, 3.84). Then $\hat{\mathcal{I}}$ yields a smooth, global cross-section of the gauge orbits in the constraint-manifold $M_{0}=\Phi^{-1}(0)$ and it satisfies

$$
\hat{\mathcal{I}}^{*}\left(\iota_{0}^{*}\left(\Omega_{M}\right)\right)=\hat{\omega}_{c}
$$

where $\iota_{0}: M_{0} \rightarrow M$ is the embedding. Therefore $\left(\hat{P}_{c}, \hat{\omega}_{c}\right)$ is a model of the reduced phase space.

Proof. The pull-back property (3.88) follows by combining Proposition 3.11 with the property $\mathcal{Z}_{x}^{*}\left(\hat{\omega}_{c}\right)=\hat{\omega}(3.70)$ and the fact that (3.86) holds. In addition, we here use that $\mathcal{Z}_{x}(\hat{P})=\hat{P}_{c}^{0}$ is dense in $\hat{P}_{c}$. We also conclude effortlessly, from (3.87) and Lemma 3.9, that the image of $\hat{\mathcal{I}}$ intersects every gauge orbit in $M_{0}$.

It only remains to show that the map $\hat{\mathcal{I}}$ is injective and no two different points on its image can be gauge equivalent. This follows if we prove the following implication:

$$
A_{[h]}(\hat{\mathcal{I}}(z, Z))=\hat{\mathcal{I}}\left(z^{\prime}, Z^{\prime}\right) \Longrightarrow h=\gamma \mathbf{1}_{n}(\gamma \in U(1)), z=z^{\prime}, Z=Z^{\prime}
$$

By proving this statement, in which $h \in G$, we shall also confirm again that $\bar{G}$ acts freely on $M_{0}$. The proof of the above implication will rely on the following properties:

1. The absolute values $|Z|,\left|z_{i}\right|$ are in one-to-one correspondence with the values of the functions $\hat{\pi}_{j}$.

2. $\vartheta_{i, i+1}(z, Z)<0$ for all $i=1, \ldots, n-1$ and depends only on the absolute values $|Z|,\left|z_{j}\right|$.

3. $\mathcal{V}_{n}(z, Z)>0$ and depends only on the absolute values of $Z$ and the $z_{j}$.

4. $\mathcal{V}_{j}(z, Z)=z_{j} f_{j}(z, Z)$ for $j=1, \ldots, n-1$, where $f_{j}(z, Z)>0$ and $f_{j}$ depends only on the absolute values of $Z$ and the $z_{k}$.

5. $\vartheta_{n, 1}(z, Z)=\bar{Z} f(z, Z)$, where $f(z, Z)>0$ and $f$ depends only on the absolute values of $Z$ and the $z_{k}$.

Property 1 is clear from (3.74), and (3.77) implies properties 3 and 4. Property 2 follows from (3.76) and property 5 from (3.79).

Now suppose that the equality

$$
A_{[h]}(\hat{\mathcal{I}}(z, Z))=\hat{\mathcal{I}}\left(z^{\prime}, Z^{\prime}\right)
$$

holds and look at its 'second component' in terms of writing the elements of $M$ as triples. This gives $h \hat{\pi}(z, Z) h^{-1}=\hat{\pi}\left(z^{\prime}, Z^{\prime}\right)$. Since the values of $\hat{\pi}$ are regular elements in the same Weyl alcove, we immediately get that $h \in \mathbb{T}(n)$ and $\hat{\pi}(z, Z)=\hat{\pi}\left(z^{\prime}, Z^{\prime}\right)$. By property 1 , this proves that $|Z|=\left|Z^{\prime}\right|$, and $\left|z_{j}\right|=\left|z_{j}^{\prime}\right|$ for all $j=1, \ldots, n-1$. By looking at the $i, i+1$ component of the equality $h \vartheta(z, Z) h^{-1}=\vartheta\left(z^{\prime}, Z^{\prime}\right)$, which follows from (3.90), we get from property 2 and the previously established facts that $h=\gamma \mathbf{1}_{n}$ for some $\gamma \in U(1)$. Therefore we must have $\xi(x, \mathcal{V}(z, Z))=\xi\left(x, \mathcal{V}\left(z^{\prime}, Z^{\prime}\right)\right)$ and this implies now by property 3 that $\mathcal{V}(z, Z)=\mathcal{V}\left(z^{\prime}, Z^{\prime}\right)$. Then property 4 entails that $z_{j}=z_{j}^{\prime}$ for all $j$, and property 5 entails that $Z=Z^{\prime}$.

Q.E.D. 
Definition 3.13. The Ruijsenaars dual of the Sutherland system of Definition 3.1 is the integrable system given by the commuting Hamiltonians generated by the spectral invariants of the unitary Lax matrix $\vartheta(z, Z)(\underline{3.73})$ on the phase space $\left(\hat{P}_{c}, \hat{\omega}_{c}\right)$ (3.67).

Note that, because of Proposition 3.11, the system introduced above is the natural completion of the rational Ruijsenaars-Schneider system that possesses the Lax matrix $\hat{L}(\hat{q}, \hat{p})(3.63)$ on the phase space $(\hat{P}, \hat{\omega})$. Here, $\hat{P}$ is identified with $\hat{P}_{c}^{0}$ (3.69). The same completion was introduced in [8] without relying on symplectic reduction.

\subsection{The duality map $\mathcal{R}$}

Let us summarize the picture that emerges from the preceding two subsections. First we recall from Section 2 that the symplectic reduction yields the phase space $\left(\left(T^{*} G\right)_{\mathrm{red}}, \Omega_{\mathrm{red}}\right)$ equipped with two Abelian algebras of integrable Hamiltonians, $\left\{H_{k}\right\}$ and $\left\{\hat{H}_{ \pm k}\right\}$ (2.18), whose flows can be obtained as projections of free flows. This statement does not refer to any coordinate system or model: the reduced phase space $\left(T^{*} G\right)_{\text {red }}$ is the space of the gauge orbits $M_{0} / \bar{G}$.

In Section 3.1 we constructed the model $(P, \omega) \equiv\left(T^{*} Q(n), \Omega_{T^{*} Q(n)}\right)$ of the reduced phase space. When regarded as functions on $P$, the family $\left\{H_{k}\right\}$ gives the commuting Sutherland Hamiltonians and the family $\left\{\hat{H}_{ \pm k}\right\}$ gives the functions of the Sutherland position-variables that represent $S(n)$-invariant trigonometric polynomials on $\mathbb{T}(n)^{0}$ (recall that $Q(n)=\mathbb{T}(n)^{0} / S(n)$ ). These latter functions separate the points of $Q(n)$, and fully determine the position data.

In Section 3.2 we constructed the model $\left(\hat{P}_{c}, \hat{\omega}_{c}\right)$ of the reduced phase space, and have taken the reduced Hamiltonians $\left\{\hat{H}_{ \pm k}\right\}$ as the commuting Hamiltonians of an integrable system, called the Ruijsenaars dual of the Sutherland system. The dual system is a completion of the rational Ruijsenaars-Schneider system characterized by the Hamiltonian $\hat{H}_{\mathrm{RS}}$ (3.64) that leaves on $\hat{P}$, which is equivalent to the dense, open submanifold $\hat{P}_{c}^{0} \subset \hat{P}_{c}(3.69)$. When viewed as functions on $\hat{P}_{c}$, the family $\left\{H_{k}\right\}$ becomes equivalent to the global position variables $\hat{\pi}_{j} \in C^{\infty}\left(\hat{P}_{c}\right)(j=1, \ldots, n)$ of the completed Ruijsenaars-Schneider system.

Our construction automatically yields a natural symplectomorphism

$$
\mathcal{R}: P \rightarrow \hat{P}_{c} \text {. }
$$

The map $\mathcal{R}$ sends the 'Sutherland representative' of a point of $\left(T^{*} G\right)_{\text {red }}$ to its 'Ruijsenaars representative'. This map operates by gauge transformations since $\hat{P}_{c}$ is realized as a global gauge slice in $M_{0}$ and $P$ is realized as the base of a sub-bundle of $M_{0}$ with finite structure group $S(n)$. The functions $\hat{\pi}_{j} \circ \mathcal{R} \in C^{\infty}(P)$ define action variables for the Sutherland system. Upon restriction to the open dense submanifold that corresponds to $M_{0}^{0} \subset M_{0}$, where the strict spectral gap condition holds, the functions $\hat{q}_{j} \circ \mathcal{Z}_{x}^{-1} \circ \mathcal{R}$ define canonical conjugates of the Sutherland actions. Conversely, when transferred to functions on $\hat{P}_{c}$, the Sutherland coordinates $q_{j}$ can be viewed as actions of the dual system. More precisely, it is the trigonometric symmetric polynomials of the functions $q_{j} \circ \mathcal{R}^{-1}$ that provide globally well-defined action variables for the dual system, because $Q(n)=\mathbb{T}(n)^{0} / S(n)$.

The above mentioned properties of the 'duality map' between $P$ and $\hat{P}_{c}$ were established originally in the impressive paper [8] on the basis of very laborious, direct arguments. In fact, our geometrically constructed map $\mathcal{R}(3.91)$ is precisely the action-angle map of [8]. This holds since our construction of the map $\mathcal{R}$ relies on the diagonalization of $J$ for the triples 
$(g, J, \xi) \in M_{0}$ in the same way as the construction of [8] relies on the diagonalization of the Sutherland Lax matrix. The link is made by means of the relation given by (3.20) with (3.10). One first obtains the identity of our $\mathcal{R}$ and the action-angle map of [8] on the dense open submanifolds of the two models of $\left(T^{*} G\right)_{\text {red }}$ corresponding to $M_{0}^{0}(3.55)$, and then it holds also globally because our embedding (3.69) of $\hat{P}$ into $\hat{P}_{c}$ is the same as the one used in [8]. The main advantage of the group theoretic approach is that the symplectic property of $\mathcal{R}$ is guaranteed automatically. One can also obtain the integration algorithms of [8] from the projections of the free flows displayed in (2.10) -(2.12). This is a routine matter, and we refrain from presenting the details. The projected free flows are complete on $\left(T^{*} G\right)_{\text {red }}$ as a result of general principles. In the present case it is readily seen that the projections of the flows (2.10) respect the strict spectral gap condition, so $M_{0}^{0} / \bar{G}$ is invariant under these flows, but the projections of the 'dual free flows' (2.10), (2.11) are complete only on the full reduced phase space $M_{0} / \bar{G}$.

\section{Coverings and dualities}

So far we have given a geometric interpretation to the lowest arrow in (1.13). The aim of this section is to expound the web of dualities and coverings for the three different versions of the Sutherland system [8] described in the Introduction and in Appendix A. The final result is represented by the diagram (4.42), which is an elaboration of the diagram (1.13).

\subsection{Discrete symmetries and coverings before KKS reduction}

To implement the ideas outlined in the Introduction, we now consider the phase spaces

$$
M_{2}:=T^{*}(\mathbb{R} \times S U(n)) \times \mathcal{O}=T^{*} \mathbb{R} \times T^{*} S U(n) \times \mathcal{O}
$$

and

$$
M_{1}:=T^{*}(U(1) \times S U(n)) \times \mathcal{O}=T^{*} U(1) \times T^{*} S U(n) \times \mathcal{O}
$$

together with $M=T^{*} U(n) \times \mathcal{O}$ that we dealt with so far. Analogously to the case of $T^{*} U(n)$, we adopt the parametrization

$$
T^{*} S U(n) \simeq S U(n) \times s u(n)=\{(\Gamma, \mathcal{J})\}, \quad \Omega_{T^{*} S U(n)}=-d \operatorname{tr}\left(\mathcal{J} d \Gamma \Gamma^{-1}\right)
$$

and also adopt the identifications $T^{*} \mathbb{R}=\mathbb{R} \times \mathbb{R}$ and $T^{*} U(1)=U(1) \times \mathbb{R}$. Therefore, the symplectic manifold $\left(M_{2}, \Omega_{M_{2}}\right)$ is realized as

$$
\begin{aligned}
& M_{2}=\mathbb{R} \times \mathbb{R} \times S U(n) \times s u(n) \times \mathcal{O}=\left\{\left(u_{0}, w_{0}, \Gamma, \mathcal{J}, \xi\right)\right\}, \\
& \Omega_{M_{2}}=d w_{0} \wedge d u_{0}-d \operatorname{tr}\left(\mathcal{J} d \Gamma \Gamma^{-1}\right)+\Omega_{\mathcal{O}}(\xi),
\end{aligned}
$$

and we also have

$$
\begin{aligned}
& M_{1}=U(1) \times \mathbb{R} \times S U(n) \times s u(n) \times \mathcal{O}=\left\{\left(\zeta_{0}, v_{0}, \Gamma, \mathcal{J}, \xi\right)\right\} \\
& \Omega_{M_{1}}=d v_{0} \wedge \frac{d \zeta_{0}}{\mathrm{i} \zeta_{0}}-d \operatorname{tr}\left(\mathcal{J} d \Gamma \Gamma^{-1}\right)+\Omega_{\mathcal{O}}(\xi)
\end{aligned}
$$


similarly to

$$
\begin{aligned}
& M=T^{*} U(n) \times \mathcal{O}=U(n) \times u(n) \times \mathcal{O}=\{(g, J, \xi)\} \\
& \Omega_{M}=-d \operatorname{tr}\left(J d g g^{-1}\right)+\Omega_{\mathcal{O}}(\xi) .
\end{aligned}
$$

There is a symplectic action of the Abelian group of the integers, $\mathbb{Z}$, on $M_{2}$ generated by the symplectic diffeomorphism $\theta(1)$ that implements the action of $1 \in \mathbb{Z}$ as follows:

$$
\theta(1):\left(u_{0}, w_{0}, \Gamma, \mathcal{J}, \xi\right) \mapsto\left(u_{0}-\frac{2 \pi}{n}, w_{0}, e^{\mathrm{i} \frac{2 \pi}{n}} \Gamma, \mathcal{J}, \xi\right) .
$$

We also consider the action of the subgroup $n \mathbb{Z}<\mathbb{Z}$ generated by

$$
\theta(1)^{n}:\left(u_{0}, w_{0}, \Gamma, \mathcal{J}, \xi\right) \mapsto\left(u_{0}-2 \pi, w_{0}, \Gamma, \mathcal{J}, \xi\right) .
$$

Moreover, a symplectic action of $\mathbb{Z}_{n}=\mathbb{Z} / n \mathbb{Z}$ (realized as the multiplicative group of the $n$-th roots of unity) on $M_{1}$ is generated by the following action of the primitive $n$-th root of unity:

$$
\alpha\left(e^{\mathrm{i} \frac{2 \pi}{n}}\right):\left(\zeta_{0}, v_{0}, \Gamma, \mathcal{J}, \xi\right) \mapsto\left(e^{-\mathrm{i} \frac{2 \pi}{n}} \zeta_{0}, v_{0}, e^{\mathrm{i} \frac{2 \pi}{n}} \Gamma, \mathcal{J}, \xi\right) .
$$

These actions arise from the cotangent lifts of corresponding actions of central subgroups of $G_{2}$ and $G_{1}(1.8)$ provided by

$$
\begin{aligned}
& \left\{\left(-k \frac{2 \pi}{n}, e^{\mathrm{i} k \frac{2 \pi}{n}} \mathbf{1}_{n}\right) \in \mathbb{R} \times S U(n) \mid k \in \mathbb{Z}\right\}<G_{2}, \\
& \left\{\left(e^{-\mathrm{i} k \frac{2 \pi}{n}}, e^{\mathrm{i} k \frac{2 \pi}{n}} \mathbf{1}_{n}\right) \in U(1) \times S U(n) \mid k=0,1, \ldots, n-1\right\}<G_{1},
\end{aligned}
$$

which are isomorphic to $\mathbb{Z}$ and $\mathbb{Z}_{n}$, respectively. The factorizations by these actions yield the identifications

$$
M_{1} \simeq M_{2} / n \mathbb{Z}, \quad M \simeq M_{2} / \mathbb{Z} \simeq\left(M_{2} / n \mathbb{Z}\right) /(\mathbb{Z} / n \mathbb{Z}) \simeq M_{1} / \mathbb{Z}_{n}
$$

The projections responsible for these identifications are given by the maps

$$
\psi_{2}: M_{2} \rightarrow M_{1}, \quad \psi_{1}: M_{1} \rightarrow M, \quad \psi:=\psi_{1} \circ \psi_{2}: M_{2} \rightarrow M,
$$

which can be written in terms of the above-introduced parametrizations as

$$
\psi_{2}:\left(u_{0}, w_{0}, \Gamma, \mathcal{J}, \xi\right) \mapsto\left(e^{\mathrm{i} u_{0}}, w_{0}, \Gamma, \mathcal{J}, \xi\right), \quad \psi_{1}:\left(\zeta_{0}, v_{0}, \Gamma, \mathcal{J}, \xi\right) \mapsto\left(\zeta_{0} \Gamma, \mathcal{J}+\frac{\mathrm{i}}{n} v_{0} \mathbf{1}_{n}, \xi\right)
$$

These are symplectic coverings, i.e.,

$$
\psi_{2}^{*}\left(\Omega_{M_{1}}\right)=\Omega_{M_{2}} \quad \text { and } \quad \psi_{1}^{*}\left(\Omega_{M}\right)=\Omega_{M_{1}} .
$$

We use these maps to pull-back the commuting families $\left\{\mathcal{H}_{k}\right\} \subset C^{\infty}(M)(2.8)$ and $\left\{\hat{\mathcal{H}}_{ \pm k}\right\} \subset$ $C^{\infty}(M)(2.9)$ to $M_{1}$ and $M_{2}$, and thereby define the respective 'free' Hamiltonians

$$
\mathcal{H}_{j}^{1}:=\mathcal{H}_{j} \circ \psi_{1}, \hat{\mathcal{H}}_{ \pm k}^{1}:=\hat{\mathcal{H}}_{ \pm k} \circ \psi_{1} \quad \text { and } \quad \mathcal{H}_{j}^{2}:=\mathcal{H}_{j} \circ \psi, \hat{\mathcal{H}}_{ \pm k}^{2}:=\hat{\mathcal{H}}_{ \pm k} \circ \psi
$$

One sees from the preceding considerations that the three phase spaces $M_{2}, M_{1}$, $M$, together with their families of 'free' Hamiltonians, are related by reductions under the respective discrete symmetries represented by the $n \mathbb{Z}$-action and the $\mathbb{Z}$-action on $M_{2}$, and the $\mathbb{Z}_{n}$-action on $M_{1}$. 


\section{2 $\bar{G}$-symmetry and KKS reduction on three levels}

In Section 3, we used the action of $\bar{G}=U(n) / U(1)$ on $M$ and analyzed the corresponding KKS type symplectic reduction. Recall that every $[y] \in \bar{G}$ can be represented by some $y \in S U(n)$, and the $\bar{G}$-action $A_{[y]}: M \rightarrow M$ and its moment map $\Phi: M \rightarrow s u(n) \simeq \operatorname{Lie}(\bar{G})^{*}$ read

$$
A_{[y]}(g, J, \xi)=\left(y g y^{-1}, y J y^{-1}, y \xi y^{-1}\right), \quad \Phi(g, J, \xi)=J-g^{-1} J g+\xi .
$$

We now lift these to corresponding $\bar{G}$-actions and moment maps on $M_{2}$ and on $M_{1}$, furnished respectively by

$$
A_{[y]}^{2}\left(u_{0}, w_{0}, \Gamma, \mathcal{J}, \xi\right)=\left(u_{0}, w_{0}, y \Gamma y^{-1}, y \mathcal{J} y^{-1}, y \xi y^{-1}\right), \quad \Phi_{2}\left(u_{0}, w_{0}, \Gamma, \mathcal{J}, \xi\right)=\mathcal{J}-\Gamma^{-1} \mathcal{J} \Gamma+\xi
$$

and

$$
A_{[y]}^{1}\left(\zeta_{0}, v_{0}, \Gamma, \mathcal{J}, \xi\right)=\left(\zeta_{0}, v_{0}, y \Gamma y^{-1}, y \mathcal{J} y^{-1}, y \xi y^{-1}\right), \quad \Phi_{1}\left(\zeta_{0}, v_{0}, \Gamma, \mathcal{J}, \xi\right)=\mathcal{J}-\Gamma^{-1} \mathcal{J} \Gamma+\xi
$$

The $\bar{G}$-actions are trivial on $T^{*} \mathbb{R}$ and on $T^{*} U(1)$, and by setting the moment maps to zero they define the reduced phase spaces

$$
\begin{gathered}
\left(T^{*} G_{2}\right)_{\mathrm{red}}:=\Phi_{2}^{-1}(0) / \bar{G}=T^{*} \mathbb{R} \times\left(T^{*} S U(n)\right)_{\mathrm{red}} \\
\left(T^{*} G_{1}\right)_{\mathrm{red}}:=\Phi_{1}^{-1}(0) / \bar{G}=T^{*} U(1) \times\left(T^{*} S U(n)\right)_{\mathrm{red}}
\end{gathered}
$$

where

$$
T^{*} S U(n)_{\mathrm{red}}:=\left(T^{*} S U(n) \times \mathcal{O}\right) / /{ }_{0} \bar{G}
$$

denotes the reduced phase space that arises from the KKS reduction of $T^{*} S U(n) \times \mathcal{O}$.

The discrete symmetries described in the previous subsection commute with the relevant $\bar{G}$-symmetries, that is, we have

$$
\begin{gathered}
A_{[y]}^{2} \circ \theta(1)=\theta(1) \circ A_{[y]}^{2}, \quad A_{[y]}^{1} \circ \alpha\left(e^{\mathrm{i} \frac{2 \pi}{n}}\right)=\alpha\left(e^{\mathrm{i} \frac{2 \pi}{n}}\right) \circ A_{[y]}^{1}, \\
\Phi_{2} \circ \theta(1)=\Phi_{2}, \quad \Phi_{1} \circ \alpha\left(e^{\mathrm{i} \frac{2 \pi}{n}}\right)=\Phi_{1} .
\end{gathered}
$$

Moreover, we also have

$$
\psi_{2} \circ A_{[y]}^{2}=A_{[y]}^{1} \circ \psi_{2}, \quad \psi_{1} \circ A_{[y]}^{1}=A_{[y]} \circ \psi_{1}, \quad \psi \circ A_{[y]}^{2}=A_{[y]} \circ \psi .
$$

The above relations allow us to conclude that it does not matter whether one first performs 'discrete reduction' (by the $\mathbb{Z}, n \mathbb{Z}$ or $\mathbb{Z}_{n}$-action) and then KKS reduction (by the $\bar{G}$-action) or the other way round, the final result will be the same. In other words, there arises a natural $\mathbb{Z}$-action (and $n \mathbb{Z}$-action) on the reduced phase space $\left(T^{*} G_{2}\right)_{\text {red }}$ and a natural $\mathbb{Z}_{n}$-action on $\left(T^{*} G_{1}\right)_{\text {red }}$, generated say by

$$
\theta(1)_{\text {red }}:\left(T^{*} G_{2}\right)_{\text {red }} \rightarrow\left(T^{*} G_{2}\right)_{\text {red }} \quad \text { and } \quad \alpha\left(e^{\mathrm{i} \frac{2 \pi}{n}}\right)_{\text {red }}:\left(T^{*} G_{1}\right)_{\text {red }} \rightarrow\left(T^{*} G_{1}\right)_{\text {red }}
$$

which induce the identifications

$$
\begin{aligned}
& \left(T^{*} G_{1}\right)_{\mathrm{red}} \simeq\left(T^{*} G_{2}\right)_{\mathrm{red}} / n \mathbb{Z} \\
& \left(T^{*} G\right)_{\mathrm{red}} \simeq\left(T^{*} G_{2}\right)_{\mathrm{red}} / \mathbb{Z}=\left(\left(T^{*} G_{2}\right)_{\mathrm{red}} / n \mathbb{Z}\right) /(\mathbb{Z} / n \mathbb{Z})=\left(T^{*} G_{1}\right)_{\mathrm{red}} / \mathbb{Z}_{n}
\end{aligned}
$$


These identifications are the remnants of those in (4.11) that survive the KKS reduction. In analogy to (4.12), the identifications are associated with certain projections

$$
\psi_{2}^{\text {red }}:\left(T^{*} G_{2}\right)_{\text {red }} \rightarrow\left(T^{*} G_{1}\right)_{\text {red }}, \quad \psi_{1}^{\text {red }}:\left(T^{*} G_{1}\right)_{\text {red }} \rightarrow\left(T^{*} G\right)_{\text {red }}, \quad \psi_{\text {red }}:=\psi_{1}^{\text {red }} \circ \psi_{2}^{\text {red }} .
$$

The phase space $\left(T^{*} G_{i}\right)_{\text {red }}(i=1,2)$ carries the two Abelian algebras of integrable Hamiltonians $\left\{H_{k}^{i}\right\}$ and $\left\{\hat{H}_{ \pm k}^{i}\right\}$ that can be characterized equivalently either as the KKS reductions of the respective free Hamiltonians in (4.15) or by means of the relations

$$
H_{j}^{1}:=H_{j} \circ \psi_{1}^{\text {red }}, \hat{H}_{ \pm k}^{1}:=\hat{H}_{ \pm k} \circ \psi_{1}^{\text {red }} \text { and } H_{j}^{2}:=H_{j} \circ \psi_{\text {red }}, \hat{H}_{ \pm k}^{2}:=\hat{H}_{ \pm k} \circ \psi_{\text {red }}
$$

To describe the projections (4.27) explicitly, we need a concrete description of the 'building block' $\left(T^{*} S U(n)\right)_{\text {red }}$ that appears as a factor both in $\left(T^{*} G_{2}\right)_{\text {red }}$ and in $\left(T^{*} G_{1}\right)_{\text {red }}$. Similarly to the $U(n)$-case, we actually have two such descriptions.

\subsection{Two models of $\left(T^{*} S U(n)\right)_{\text {red }}$ in duality}

Referring to the notations of Appendix A.1, consider the cotangent bundle

$$
T^{*} S Q(n) \simeq T^{*} \operatorname{Simp}_{n-1} \simeq \operatorname{Simp}_{n-1} \times \mathbb{R}^{n-1}=\{(\delta, \gamma)\}, \quad \Omega_{T^{*} S Q(n)}=\sum_{j=1}^{n-1} d \gamma_{j} \wedge d \delta_{j}
$$

By using $\beta_{k}(\delta)$ in (A.15) and $\beta(\delta):=\operatorname{diag}\left(\beta_{1}(\delta), \ldots, \beta_{n}(\delta)\right)$ for $\delta \in \operatorname{Simp}_{n-1}$ (A.5), introduce the $s u(n)$-valued function

$$
\mathcal{J}(\delta, \gamma):=\mathrm{i} \sum_{j=1}^{n-1} \gamma_{j}\left(E_{j, j}-E_{j+1, j+1}\right)+\mathrm{i} x \sum_{a \neq b} \frac{E_{a, b}}{1-e^{\mathrm{i}\left(\beta_{b}(\delta)-\beta_{a}(\delta)\right)}} .
$$

By setting $\xi_{0}:=-\mathrm{i} x \sum_{a \neq b} E_{a, b}$, the manifold

$$
\left(T^{*} S U(n)\right)_{\mathrm{red}}^{\mathrm{I}}:=\left\{\left(e^{\mathrm{i} \beta(\delta)}, \mathcal{J}(\delta, \gamma), \xi_{0}\right) \mid(\delta, \gamma) \in T^{*} \operatorname{Simp}_{n-1}\right\} \simeq T^{*} S Q(n)
$$

is a model of the reduced phase space defined by (4.21). In fact, $\left(T^{*} S U(n)\right)_{\mathrm{red}}^{\mathrm{I}} \subset T^{*} S U(n) \times \mathcal{O}$ is a global cross-section for the action of $\bar{G}$ on the zero level set of the moment map $\Phi_{0}$ : $T^{*} S U(n) \times \mathcal{O} \rightarrow s u(n)$ given by

$$
\Phi_{0}(\Gamma, \mathcal{J}, \xi)=\mathcal{J}-\Gamma^{-1} \mathcal{J} \Gamma+\xi
$$

and the pull-back of the symplectic form of $T^{*} S U(n) \times \mathcal{O}$ on $\left(T^{*} S U(n)\right)_{\text {red }}^{\mathrm{I}}$ coincides with $\Omega_{T^{*} S Q(n)}$. The proof of this result [12] follows the lines of Section 3.1. The model (4.31) of $\left(T^{*} S U(n)\right)_{\text {red }}$ is naturally associated with the 'relative motion' (i.e., motion in the center of mass frame) of the $n$ distinguished particles on the circle; see also Appendix A. The relative motion is governed by the Hamiltonian $-\frac{1}{2} \operatorname{tr}\left(\mathcal{J}(\delta, \gamma)^{2}\right)$, which is Liouville integrable on account of the commuting family given by the spectral invariants of $\mathcal{J}(\delta, \gamma)$.

Our next goal is to identify a dual model of the reduced phase space $\left(T^{*} S U(n)\right)_{\text {red }}$ with

$$
\mathbb{C}^{n-1}=\left\{\zeta=\left(\zeta_{1}, \ldots, \zeta_{n-1}\right)\right\}, \quad \Omega_{\mathbb{C}^{n-1}}:=\mathrm{i} \sum_{j=1}^{n-1} d \zeta_{j} \wedge d \bar{\zeta}_{j}
$$


which can be recast equivalently as the standard symplectic vector space of $\mathbb{R}^{2(n-1)}$. For this purpose, by a suitable modification of the formulas of Section 3.2, we construct a second global cross-section in $\Phi_{0}^{-1}(0) \subset T^{*} S U(n) \times \mathcal{O}$ as follows. First, we define the functions

$$
\hat{\pi}_{k}^{0}(\zeta):=x \frac{n+1-2 k}{2}-\sum_{1 \leq j \leq(k-1)} \frac{j}{n}\left|\zeta_{j}\right|^{2}+\sum_{k \leq j \leq(n-1)} \frac{n-j}{n}\left|\zeta_{j}\right|^{2}, \quad \forall k=1, \ldots, n,
$$

so that we have $\sum_{k=1}^{n} \hat{\pi}_{k}^{0}(\zeta)=0$. Second, we introduce the auxiliary functions $Q_{j, k}^{0}(\zeta)$ by replacing $\hat{\pi}$ in (3.75) with $\hat{\pi}^{0}$. Third, we define the $S U(n)$-valued function $\vartheta^{0}(\zeta)$ by means of the replacements

$$
Z \rightarrow 1, \quad z_{j} \rightarrow \zeta_{j}, \quad \hat{\pi}_{j} \rightarrow \hat{\pi}_{j}^{0}, \quad Q_{j, k} \rightarrow Q_{j, k}^{0}
$$

in the formulas (3.78)-(3.84), and analogously define the $\mathbb{C}^{n}$-valued function $\mathcal{V}^{0}(\zeta)$ by modifying (3.77). Finally, similarly to the formula (3.85), we define the smooth map

$$
\hat{\mathcal{I}}^{0}: \mathbb{C}^{n-1} \rightarrow \Phi_{0}^{-1}(0) \subset T^{*} S U(n) \times \mathcal{O}, \quad \hat{\mathcal{I}}^{0}(\zeta):=\left(\vartheta^{0}(\zeta)^{-1},-\mathrm{i} \hat{\pi}^{0}(\zeta), \xi\left(x, \mathcal{V}^{0}(\zeta)\right)\right)
$$

It can be shown that $\hat{\mathcal{I}}^{0}$ is injective and its image,

$$
\left(T^{*} S U(n)\right)_{\mathrm{red}}^{\mathrm{II}}:=\left\{\left(\vartheta^{0}(\zeta)^{-1},-\mathrm{i} \hat{\pi}^{0}(\zeta), \xi\left(x, \mathcal{V}^{0}(\zeta)\right)\right) \mid \zeta \in \mathbb{C}^{n-1}\right\} \simeq \mathbb{C}^{n-1},
$$

is a global cross-section of the $\bar{G}$-orbits that converts the reduced symplectic form into $\Omega_{\mathbb{C}^{n-1}}$. Of course, this model of the reduced phase space carries the distinguished commuting Hamiltonians provided by the spectral invariants of $\vartheta^{0}(\zeta)$.

The above construction yields automatically the 'duality symplectomorphism in the center of mass frame'

$$
\mathcal{R}_{0}:\left(T^{*} S U(n)\right)_{\text {red }}^{\mathrm{I}} \rightarrow\left(T^{*} S U(n)\right)_{\text {red }}^{\mathrm{II}},
$$

which operates by the pertinent gauge transformations between the two global cross-sections. The map $\mathcal{R}_{0}$ can be viewed as an action-angle transform for the center of mass version of the Sutherland system, and its inverse is an action-angle transform for the $S U(n)$-version of the completed dual Ruijsenaars-Schneider system.

\subsection{The web of discrete reductions and dualities}

The preceding constructions imply that the dual phase spaces associated with $U(n)$,

$$
P \simeq\left(T^{*} G\right)_{\mathrm{red}} \simeq \hat{P}_{c}
$$

possess the symplectic covering spaces given by the dual pairs

$$
T^{*} U(1) \times\left(T^{*} S U(n)\right)_{\mathrm{red}}^{\mathrm{I}} \simeq\left(T^{*} G_{1}\right)_{\mathrm{red}} \simeq T^{*} U(1) \times\left(T^{*} S U(n)\right)_{\mathrm{red}}^{\mathrm{II}}
$$

and

$$
T^{*} \mathbb{R} \times\left(T^{*} S U(n)\right)_{\mathrm{red}}^{\mathrm{I}} \simeq\left(T^{*} G_{2}\right)_{\mathrm{red}} \simeq T^{*} \mathbb{R} \times\left(T^{*} S U(n)\right)_{\mathrm{red}}^{\mathrm{II}} .
$$

The respective dual pairs appear on the two ends of the three chains of symplectomorphisms in (4.39)-(4.41), while the middle term (such as $\left(T^{*} G\right)_{\text {red }}$ etc) refers to the 'abstract reduced phase space' that exists as a space of orbits, irrespective of any model of it. The dual systems 
of integrable Hamiltonians are provided by $\left\{H_{k}^{i}\right\}$ and $\left\{\hat{H}_{ \pm k}^{i}\right\}$ (4.28) expressed in terms of the alternative models of $\left(T^{*} G_{i}\right)_{\text {red }}$ in analogy to the Hamiltonians $\left\{H_{k}\right\}$ and $\left\{\hat{H}_{ \pm k}\right\}$ on $\left(T^{*} G\right)_{\text {red }}$. In more detail, the situation is depicted by the following commutative diagram:

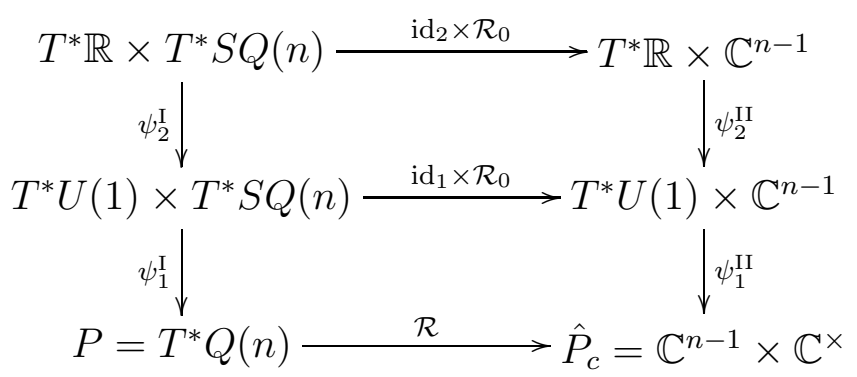

This diagram is the detailed version of the diagram (1.13) presented the Introduction, where now we have a rather complete understanding of all its ingredients. In particular, $\psi_{2}^{\mathrm{I}}$ in (4.42) represents the projection $\psi_{2}^{\text {red }}(4.27)$ of the $n \mathbb{Z}$-reduction in terms of the models

$$
\begin{aligned}
& \left(T^{*} G_{2}\right)_{\mathrm{red}} \simeq T^{*} \mathbb{R} \times\left(T^{*} S U(n)\right)_{\mathrm{red}}^{\mathrm{I}} \equiv T^{*} \mathbb{R} \times T^{*} S Q(n), \\
& \left(T^{*} G_{1}\right)_{\mathrm{red}} \simeq T^{*} U(1) \times\left(T^{*} S U(n)\right)_{\mathrm{red}}^{\mathrm{I}} \equiv T^{*} U(1) \times T^{*} S Q(n),
\end{aligned}
$$

and $\psi_{2}^{\mathrm{II}}$ represent $\psi_{2}^{\text {red }}$ in terms of the models

$$
\begin{aligned}
& \left(T^{*} G_{2}\right)_{\mathrm{red}} \simeq T^{*} \mathbb{R} \times\left(T^{*} S U(n)\right)_{\mathrm{red}}^{\mathrm{II}} \equiv T^{*} \mathbb{R} \times \mathbb{C}^{n-1} \\
& \left(T^{*} G_{1}\right)_{\mathrm{red}} \simeq T^{*} U(1) \times\left(T^{*} S U(n)\right)_{\mathrm{red}}^{\mathrm{II}} \equiv T^{*} U(1) \times \mathbb{C}^{n-1} .
\end{aligned}
$$

The map $\mathcal{R}_{0}$ is the 'duality symplectomorphism in the center of mass frame' (4.38), while $\mathrm{id}_{2}$ and $\mathrm{id}_{1}$ denote the identity maps of $T^{*} \mathbb{R}$ and $T^{*} U(1)$, respectively. The maps $\psi_{2}^{\mathrm{I}}$ and $\psi_{2}^{\mathrm{II}}$ are quite simple, since they have factorized form and their non-trivial factor is the obvious $\operatorname{map} T^{*} \mathbb{R} \rightarrow T^{*} U(1)$ that sends $(u, w)$ to $\left(\zeta_{0}, v_{0}\right):=\left(e^{\mathrm{i} u}, w\right)$. The maps $\psi_{1}^{\mathrm{I}}$ and $\psi_{1}^{\mathrm{II}}$ are the projections induced by the $\mathbb{Z}_{n}$-action, $\alpha\left(e^{\mathrm{i} \frac{2 \pi}{n}}\right)_{\text {red }}:\left(T^{*} G_{1}\right)_{\text {red }} \rightarrow\left(T^{*} G_{1}\right)_{\text {red }}$, that admits the alternative realizations

$$
\begin{aligned}
& \alpha\left(e^{\mathrm{i} \frac{2 \pi}{n}}\right)_{\text {red }}^{\mathrm{I}}: T^{*} U(1) \times T^{*} S Q(n) \rightarrow T^{*} U(1) \times T^{*} S Q(n), \\
& \alpha\left(e^{\mathrm{i} \frac{2 \pi}{n}}\right)_{\text {red }}^{\mathrm{I}}:\left(\zeta_{0}, v_{0}, \delta, \gamma\right) \mapsto\left(e^{-\mathrm{i} \frac{2 \pi}{n}} \zeta_{0}, v_{0}, \delta_{2}, \ldots, \delta_{n-1}, 2 \pi-\sum_{j=1}^{n-1} \delta_{j}, \gamma_{2}-\gamma_{1}, \ldots, \gamma_{n-1}-\gamma_{1},-\gamma_{1}\right),
\end{aligned}
$$

and

$$
\begin{aligned}
& \alpha\left(e^{\mathrm{i} \frac{2 \pi}{n}}\right)_{\mathrm{red}}^{\mathrm{II}}: T^{*} U(1) \times \mathbb{C}^{n-1} \rightarrow T^{*} U(1) \times \mathbb{C}^{n-1} \\
& \alpha\left(e^{\mathrm{i} \frac{2 \pi}{n}}\right)_{\mathrm{red}}^{\mathrm{II}}:\left(\zeta_{0}, v_{0},\left\{\zeta_{j}\right\}_{j=1}^{n-1}\right) \mapsto\left(e^{-\mathrm{i} \frac{2 \pi}{n}} \zeta_{0}, v_{0},\left\{e^{\mathrm{i} \frac{2 \pi}{n}(n-j)} \zeta_{j}\right\}_{j=1}^{n-1}\right) .
\end{aligned}
$$

These formulas can be obtained directly from the definitions by 'diagram chasing', and agree with corresponding formulae in [8]. The associated formula of $\psi_{1}^{\mathrm{II}}$ is found to be

$$
\psi_{1}^{\mathrm{II}}:\left(\zeta_{0}, v_{0}, \zeta\right) \mapsto(z, Z) \text { with } z_{j}=\zeta_{0}^{n-j} \zeta_{j}, Z=\zeta_{0}^{n} \exp \left(\frac{1-n}{2} x+\frac{v_{0}}{n}+\sum_{k=1}^{n-1} \frac{k-n}{n}\left|\zeta_{k}\right|^{2}\right)
$$

and $\psi_{1}^{\mathrm{I}}$ is described in Appendix A.3. 
We constructed the duality map $\mathcal{R}$ in Section 3, and now we observe that $\mathcal{R}$ can be characterized as the unique map that makes the diagram (4.42) commute. All the maps that appear in (4.42) can be found ${ }^{3}$ in the paper [8], too, and there the investigation of the properties of $\mathcal{R}$ was actually based on a corresponding commutative diagram. Our geometric derivation reveals the natural group theoretic origin of the web of dualities and coverings encoded by (4.42).

\section{Discussion}

In this paper we presented a group theoretic interpretation of the duality relation between the trigonometric Sutherland system and the completion of a certain real form of the complex rational Ruijsenaars-Schneider system. More precisely, we dealt with three variants of these dual pairs and connected their covering Poisson maps to the covering homomorphisms in (1.8). All ingredients of our diagram (4.42) were constructed previously by Ruijsenaars [8] relying on direct methods, i.e., without using symplectic reduction. The powerful tool of symplectic reduction allowed us to shed a new light on the web of dualities and coverings, and it also allowed us to simplify the original arguments of [8]. In particular, the symplectic character of the duality maps is obvious in our setting, while originally this required a complicated proof.

As was briefly mentioned also in the Introduction, three other cases of Ruijsenaars' duality relations [6, 8] were treated before by reductions of finite-dimensional, real symplectic manifolds. First, one can explain the self-duality of the rational Calogero system with the help of the classical KKS reduction [12] of $T^{*} u(n)$. Second, one can obtain [20] the duality between the standard hyperbolic Sutherland and rational Ruijsenaars-Schneider systems by reduction of the phase space $T^{*} \mathcal{P}(n)$, where $\mathcal{P}(n)$ is the symmetric space of positive definite Hermitian matrices. Third, the dual pair involving the standard trigonometric Ruijsenaars-Schneider system can be interpreted in terms of a Poisson-Lie analogue of the KKS reduction [21]. In the last case the unreduced phase space is the Heisenberg double of the Poisson-Lie group $U(n)$. This PoissonLie analogue of $T^{*} U(n)$ is the real Lie group $G L(n, \mathbb{C})$ equipped with a certain symplectic structure. The 'relativistic' generalization [8] of the diagram (4.42) can also be obtained by reducing covering spaces of $G L(n, \mathbb{C})$.

However, at the moment of writing, there still exist two cases of the duality established in [8] for which an interpretation of the above type is not known. These concern the self-dual systems provided on the one hand by the standard, physically most important, hyperbolic RuijsenaarsSchneider system, and on the other by the so-called $\mathrm{III}_{\mathrm{b}}$ real form of the complex trigonometric Ruijsenaars-Schneider system [8]. Motivated by the gross features of these systems, we find it tempting to speculate that they should be the reductions of suitable $U(n)$-symmetric 'free systems' on manifolds of the form

$$
\mathcal{P}(n) \times \mathcal{P}(n) \text { and } \quad U(n) \times U(n) .
$$

Specifically, we expect that the III $_{\mathrm{b}}$ real form (also known as the compactified trigonometric system [24]) can be derived by applying q-Hamiltonian reduction to $U(n) \times U(n)$ equipped with the structure of the fused double defined in [25], and this will yield the correct finite-dimensional

\footnotetext{
${ }^{3}$ In continuation of the footnote given after (1.13), we remark that the symbols $T^{*} S Q(n), \mathbb{C}^{n-1}$ and $\mathcal{R}_{0}$ in (4.42) correspond respectively to the symbols $M, \hat{M}$ and $\phi$ in (1.74) of [8]. Note also that in [8] the transposition of the two components of $\mathbb{R}^{2}$ is used in place of our $\mathrm{id}_{2}$ in (4.42) due to an immaterial difference of conventions.
} 
counterpart of the infinite-dimensional reduction suggested in [13]. This issue is currently under investigation. It is a very intriguing question whether it is possible to construct a suitable $U(n)$-symmetric 'free system' on $\mathcal{P}(n) \times \mathcal{P}(n)$ so that it could serve as the starting point for the derivation of the hyperbolic Ruijsenaars-Schneider system by reduction. We stress that these questions concern systems with real particle-positions, and hence their solution requires to go beyond the treatment of the complex trigonometric system presented in [17, 26].

It is also an open problem to extend the Ruijsenaars dualities to systems with two types of particles as well as to $B C(n)$ systems. In the former case the action-angle maps, without an interpretation in terms of dualities, were described in [7]. In our opinion these problems are important and non-trivial. They could pose a worthwhile challenge for the interested reader.

\section{A Three variants of the Sutherland phase space}

In this appendix we explain how the phase spaces $P_{2}, P_{1}$ and $P$ displayed in (1.4) correspond, respectively, to distinguishable particles moving on the line $\mathbb{R}$ or on the circle $U(1)$, or to indistinguishable particles on the circle. Our treatment here is close to [8], but we pay more attention to the Lie-theoretic interpretation of the pertinent configuration spaces.

\section{A.1 Distinguishable particles on the line}

Consider $n$ distinguishable particles on the line interacting according to the Hamiltonian of the form (1.1). Due to the repulsive periodic potential, the order of the particles cannot change during the motion and the distance between the particles is also bounded. One possible choice from equivalent configuration spaces is thus the convex domain

$$
C(n):=\left\{u \in \mathbb{R}^{n} \mid u_{1}>u_{2}>\cdots>u_{n}, u_{1}-u_{n}<2 \pi\right\} .
$$

The corresponding phase space,

$$
T^{*} C(n)=C(n) \times \mathbb{R}^{n}=\left\{(u, w) \mid u \in C(n), w \in \mathbb{R}^{n}\right\}, \quad \Omega_{T^{*} C(n)}=\sum_{j=1}^{n} d w_{j} \wedge d u_{j},
$$

is equipped with the Hamiltonian

$$
H_{\text {Suth }}^{T^{*} C(n)}(u, w)=\frac{1}{2} \sum_{j=1}^{n} w_{j}^{2}+\frac{1}{4} \sum_{1 \leq i<j \leq n} \frac{x^{2}}{\sin ^{2}\left(\frac{u_{i}-u_{j}}{2}\right)} .
$$

The configuration space $C(n)$ can be represented as the Cartesian product,

$$
C(n) \simeq \mathbb{R} \times \operatorname{Simp}_{n-1},
$$

where the line $\mathbb{R}$ belongs to the center of mass motion, and the open simplex,

$$
\operatorname{Simp}_{n-1}:=\left\{\delta \in \mathbb{R}^{n-1} \mid \delta_{j}>0, \quad \sum_{j=1}^{n-1} \delta_{j}<2 \pi\right\}
$$


is the configuration space of the relative motion. We denote the center of mass coordinate by $u_{0}$, its canonical conjugate by $w_{0}$, and the canonical conjugates of the relative coordinates $\delta_{j}$ by $\gamma_{j}$. With these notations, the 'separated form' of the phase space is

$$
T^{*} C(n)=T^{*} \mathbb{R} \times T^{*} \operatorname{Simp}_{n-1} \equiv(\mathbb{R} \times \mathbb{R}) \times\left(\operatorname{Simp}_{n-1} \times \mathbb{R}^{n-1}\right)=\left\{\left(u_{0}, w_{0}\right)\right\} \times\{(\delta, \gamma)\}
$$

with the symplectic form

$$
\Omega_{T^{*} C(n)}=\Omega_{T^{*} \mathbb{R}}+\Omega_{T^{*} \operatorname{Simp}_{n-1}}=d w_{0} \wedge d u_{0}+\sum_{j=1}^{n-1} d \gamma_{j} \wedge d \delta_{j}
$$

The map between the above two systems of Darboux coordinates on $T^{*} C(n)$ is provided by the following formulae:

$$
\begin{aligned}
\delta_{j} & =u_{j}-u_{j+1}, & \gamma_{j} & =\sum_{k=1}^{j} w_{k}-\frac{j}{n} \sum_{k=1}^{n} w_{k}, \quad j=1, \ldots, n-1, \\
u_{0} & =\frac{1}{n} \sum_{k=1}^{n} u_{k}, & w_{0} & =\sum_{k=1}^{n} w_{k} .
\end{aligned}
$$

The inverse formulae are

$$
\begin{aligned}
& u_{n}=u_{0}-\frac{1}{n} \sum_{k=1}^{n-1} k \delta_{k}, \quad u_{j}=u_{0}-\frac{1}{n} \sum_{k=1}^{n-1} k \delta_{k}+\sum_{k=j}^{n-1} \delta_{k}, \quad j=1, \ldots, n-1, \\
& w_{m}=\gamma_{m}-\gamma_{m-1}+\frac{1}{n} w_{0}, \quad m=1, \ldots, n, \quad\left(\gamma_{0}=\gamma_{n}:=0\right) .
\end{aligned}
$$

When expressed in terms of the 'separated variables', the kinetic energy becomes

$$
\frac{1}{2} \sum_{k=1}^{n} w_{k}^{2}=\frac{1}{2 n} w_{0}^{2}+\frac{1}{2} \sum_{j, k=1}^{n-1} A_{j, k} \gamma_{j} \gamma_{k}
$$

where

$$
A_{j, k}:=\operatorname{tr}\left(\left(E_{j, j}-E_{j+1, j+1}\right)\left(E_{k, k}-E_{k+1, k+1}\right)\right)
$$

is the Cartan matrix of $\operatorname{sl}(n)$; the potential energy depends only on the relative coordinates $\delta_{j}$.

For our purposes, it is important to note that $\operatorname{Simp}_{n-1}$ can be regarded as a model of the group theoretically natural configuration space

$$
S Q(n):=S \mathbb{T}(n)^{0} / S(n),
$$

which is the space of Weyl orbits in the regular part of the maximal torus $S \mathbb{T}(n)<S U(n)$. To explain this, introduce the open Weyl alcove

$$
\mathcal{A}(n-1):=\left\{\beta=\operatorname{diag}\left(\beta_{1}, \beta_{2}, \ldots, \beta_{n}\right) \mid \beta_{1}>\beta_{2}>\cdots>\beta_{n}, \beta_{1}-\beta_{n}<2 \pi, \operatorname{tr}(\beta)=0\right\} .
$$

The exponential map can be used to map $\mathcal{A}(n-1)$ diffeomorphically onto the open submanifold

$$
A(n-1):=\left\{e^{\mathrm{i} \beta} \mid \beta \in \mathcal{A}(n-1)\right\} \subset S T(n)^{0},
$$


which is a fundamental domain for the $S(n)$-action on $S \mathbb{T}(n)^{0}$. Moreover, we define a diffeomorphism between $\mathcal{A}(n-1)$ and the simplex $\operatorname{Simp}_{n-1}$ by the map $\delta \mapsto \beta(\delta)$ given by

$$
\beta_{n}(\delta)=-\frac{1}{n} \sum_{k=1}^{n-1} k \delta_{k}, \quad \beta_{j}(\delta)=\beta_{n}(\delta)+\sum_{k=j}^{n-1} \delta_{k}, \quad j=1, \ldots, n-1 .
$$

Altogether we have the identifications

$$
S Q(n) \longleftrightarrow A(n-1) \longleftrightarrow \mathcal{A}(n-1) \longleftrightarrow \operatorname{Simp}_{n-1} .
$$

\section{A.2 Distinguishable particles on the circle}

The manifold $\mathbb{T}(n)^{0}(\underline{3.2})$ can be viewed as the set of possible configurations of $n$ distinguishable 'non-coinciding point particles' moving on the unit circle $U(1)$. We attach the labels $1, \ldots, n$ to the distinguished particles and identify $\tau=\operatorname{diag}\left(\tau_{1}, \ldots, \tau_{n}\right) \in \mathbb{T}(n)^{0}$ as the configuration for which $\tau_{k}$ is the location of the particle with label $k$. It is easy to see that the connected components of the manifold $\mathbb{T}(n)^{0}$ correspond to the different possible cyclic orderings of the $n$ distinct particles. The cyclic orderings correspond, in turn, to the $(n-1)$ ! different $n$-cycles in the group $S(n)$. We can restrict the dynamics to a single connected component, and we choose the particular one given by the manifold

$$
K(n):=\left\{e^{\mathrm{i} q} \mid q=\operatorname{diag}\left(q_{1}, \ldots, q_{n}\right), q_{1}>q_{2}>\cdots>q_{n}, q_{1}-q_{n}<2 \pi\right\} .
$$

That is, we take the phase space of the distinguished particles to be the symplectic manifold

$$
T^{*} K(n)=K(n) \times \mathbb{R}^{n}=\left\{\left(e^{\mathrm{i} q}, p\right)\right\}, \quad \Omega_{T^{*} K(n)}=\sum_{k=1}^{n} d p_{k} \wedge \frac{d e^{\mathrm{i} q_{k}}}{\mathrm{i} e^{\mathrm{i} q_{k}}} .
$$

It should be noted that the variable $q_{n}$ is ambiguous up to multiples of $2 \pi$, but $e^{\mathrm{i} q_{n}}$ is welldefined and once $q_{n}$ is chosen then the other $q_{j}$ are uniquely determined by the conditions specified in (A.17). Taking this into account, globally well-defined smooth coordinates on $K(n)$ are provided by

$$
\zeta_{0}:=e^{\mathrm{i} q_{n}} \exp \left(\frac{\mathrm{i}}{n} \sum_{j=1}^{n-1} j\left(q_{j}-q_{j+1}\right)\right) \quad \text { and } \quad \delta_{j}:=q_{j}-q_{j+1}, \quad j=1, \ldots, n-1 .
$$

These formulae define the map $K(n) \rightarrow U(1) \times \operatorname{Simp}_{n-1}$,

$$
e^{\mathrm{i} q} \mapsto\left(\zeta_{0}\left(e^{\mathrm{i} q}\right), \delta\left(e^{\mathrm{i} q}\right)\right)
$$

This is a diffeomorphism with the inverse map $U(1) \times \operatorname{Simp}_{n-1} \rightarrow K(n)$ furnished by

$$
\left(\zeta_{0}, \delta\right) \mapsto \zeta_{0} e^{\mathrm{i} \beta(\delta)}
$$

where $\beta_{k}(\delta)$ is given by (A.15). As a consequence, we obtain the identification

$$
T^{*} K(n)=T^{*} U(1) \times T^{*} \operatorname{Simp}_{n-1} \equiv(U(1) \times \mathbb{R}) \times\left(\operatorname{Simp}_{n-1} \times \mathbb{R}^{n-1}\right)=\left\{\left(\zeta_{0}, v_{0}\right)\right\} \times\{(\delta, \gamma)\}
$$


whereby we can write the symplectic form as

$$
\Omega_{T^{*} K(n)} \simeq \Omega_{T^{*} U(1)}+\Omega_{T^{*} \operatorname{Simp}_{n-1}}=d v_{0} \wedge \frac{d \zeta_{0}}{\mathrm{i} \zeta_{0}}+\sum_{j=1}^{n-1} d \gamma_{j} \wedge d \delta_{j}
$$

The canonical momenta $v_{0}$ and $\gamma_{j}$ are related to the momenta $p_{k}$ of the individual particles according to

$$
v_{0}=\sum_{k=1}^{n} p_{k}, \quad \gamma_{j}=\sum_{k=1}^{j} p_{k}-\frac{j}{n} \sum_{k=1}^{n} p_{k} .
$$

By using the pertinent identification in (A.16), the second term in (A.23) can be regarded also as the symplectic form of $T^{*} S Q(n) \simeq T^{*} \operatorname{Simp}_{n-1}$.

Now some remarks are in order. First, note that $\zeta_{0}($ A.19) defines a 'center of mass' for the distinguishable particles moving on the circle. In fact, $\zeta_{0}$ gets rotated by the angle $\alpha$ if all the particle positions are rigidly rotated by the same angle $\alpha$; and the relative coordinates $\delta_{j}$ do not change under these rigid rotations.

Second, let us notice that the phase space of the particles on the circle is actually a symplectic quotient of the phase space of the particles on the line. Indeed, there is a free, properly discontinuous, symplectic action of the group $n \mathbb{Z}<\mathbb{Z}$ on $T^{*} C(n)$ generated by the action of $n \in n \mathbb{Z}$ defined by $\left(\left(u_{1}, \ldots, u_{n}\right), w\right) \mapsto\left(\left(u_{1}-2 \pi, \ldots, u_{n}-2 \pi\right), w\right)$, which translates all particle coordinates by $2 \pi$. Equivalently, it acts on the separated variables in (A.6) according to $\left(u_{0}, w_{0}, \delta, \gamma\right) \mapsto\left(u_{0}-2 \pi, w_{0}, \delta, \gamma\right)$. The corresponding space of orbits is naturally a symplectic manifold, and we can make the identification

$$
T^{*} C(n) / n \mathbb{Z} \equiv T^{*} K(n)
$$

The associated projection

$$
\psi_{2}^{\mathrm{I}}: T^{*} C(n) \rightarrow T^{*} K(n)
$$

is given explicitly as

$$
(u, w) \mapsto\left(e^{\mathrm{i} q}, p\right):=\left(e^{\mathrm{i} \operatorname{diag}\left(u_{1}, \ldots, u_{n}\right)}, w\right),
$$

or in terms of the separated variables displayed in (A.6) and (A.22) simply as

$$
T^{*} \mathbb{R} \times T^{*} \operatorname{Simp}_{n-1} \ni\left(u_{0}, w_{0}, \delta, \gamma\right) \mapsto\left(e^{\mathrm{i} u_{0}}, w_{0}, \delta, \gamma\right) \in T^{*} U(1) \times T^{*} \operatorname{Simp}_{n-1}
$$

Thus the only effect of the factorization by the $n \mathbb{Z}$-action is to identify the variable $\zeta_{0} \in U(1)$ as the exponential of the center of mass $u_{0}$ of the particles on the line. The projection $\psi_{2}^{\mathrm{I}}$ is locally symplectic, and $T^{*} C(n)$ is a symplectic $n \mathbb{Z}$-covering of $T^{*} K(n)$. On account of (A.16), the notation $\psi_{2}^{\mathrm{I}}(\underline{\mathrm{A} .26})$ is consistent with the diagram (4.42).

\section{A.3 Indistinguishable particles on the circle}

The permutation group $S(n)$ acts freely on $\mathbb{T}(n)^{0}$ by the formula

$$
\sigma(\tau)_{k}:=\tau_{\sigma^{-1}(k)}, \quad \forall \sigma \in S(n), \forall \tau=\operatorname{diag}\left(\tau_{1}, \ldots, \tau_{n}\right) \in \mathbb{T}(n)^{0} .
$$

By definition, the configuration space $Q(n)$ is obtained from $\mathbb{T}(n)^{0}$ by identifying the elements that are related by permutations. Thus $Q(n)$ may describe indistinguishable particles, or 
distinct particles whose distinction is erased when recording the configurations. It is clear that every element of $\mathbb{T}(n)^{0}$ can brought into the connected component $K(n) \subset \mathbb{T}(n)^{0}$ A.17) by a suitable permutation, and the subgroup of $S(n)$ that maps $K(n)$ to $K(n)$ is generated by the cyclic permutation, $\mu \in S(n)$ given by $\mu:(1,2, \ldots, n) \mapsto(\mu(1), \mu(2), \ldots, \mu(n)):=$ $(n, 1, \ldots, n-1)$. Denoting this subgroup as $\mathbb{Z}_{n}<S(n)$, we obtain the identification

$$
Q(n)=\mathbb{T}(n)^{0} / S(n)=K(n) / \mathbb{Z}_{n} .
$$

In terms of the model $K(n) \equiv U(1) \times \operatorname{Simp}_{n-1}$ given by $(\underline{A .20})$, the cyclic permutation acts as

$$
\mu:\left(\zeta_{0}, \delta_{1}, \ldots, \delta_{n-2}, \delta_{n-1}\right) \mapsto\left(e^{-\mathrm{i} \frac{2 \pi}{n}} \zeta_{0}, \delta_{2}, \ldots, \delta_{n-1}, 2 \pi-\sum_{j=1}^{n-1} \delta_{j}\right)
$$

and the cotangent lift of this action reads

$$
\mu:\left(\zeta_{0}, v_{0}, \delta, \gamma\right) \mapsto\left(e^{-\mathrm{i} \frac{2 \pi}{n}} \zeta_{0}, v_{0}, \delta_{2}, \ldots, \delta_{n-1}, 2 \pi-\sum_{j=1}^{n-1} \delta_{j}, \gamma_{2}-\gamma_{1}, \ldots, \gamma_{n-1}-\gamma_{1},-\gamma_{1}\right)
$$

If, by using that $\operatorname{Simp}_{n-1} \simeq S Q(n)$ according to (A.16), we identify $K(n)$ with $U(1) \times S Q(n)$ and denote the $S(n)$-orbit of any $\tau \in \mathbb{T}(n)^{0}$ by $[\tau]$, then the action (A.31) takes the form

$$
\mu:\left(\zeta_{0},[\tau]\right) \mapsto\left(e^{-\mathrm{i} \frac{2 \pi}{n}} \zeta_{0},\left[e^{\mathrm{i} \frac{2 \pi}{n}} \tau\right]\right) .
$$

In this picture the projection $K(n) \rightarrow Q(n)$ associated with (A.30) can be written simply as

$$
U(1) \times S Q(n) \ni\left(\zeta_{0},[\tau]\right) \mapsto\left[\zeta_{0} \tau\right] \in Q(n) .
$$

The cotangent lift of this projection yields the map

$$
\psi_{1}^{\mathrm{I}}: T^{*} K(n) \simeq T^{*} U(1) \times T^{*} S Q(n) \rightarrow T^{*} Q(n),
$$

whereby $\left(\psi_{1}^{\mathrm{I}}\right)^{*}\left(\Omega_{T^{*} Q(n)}\right)=\Omega_{T^{*} K(n)}$. The notation $\psi_{1}^{\mathrm{I}}$ conforms with diagram (4.42). Since $T^{*} K(n)$ is a $\mathbb{Z}_{n}$ symplectic covering of $T^{*} Q(n)$, one can study the dynamics on $T^{*} Q(n)$ either by working on $T^{*} K(n)$ and then projecting to $T^{*} Q(n)$, or by directly working on the non-trivial manifold $Q(n)$. In the latter approach one may use the coordinates introduced below.

\section{A.4 Convenient coordinates on $Q(n)$}

We here construct a cover of $Q(n)$ by two contractible coordinate charts. For this purpose, we regard the elements of $Q(n)$ as $S(n)$-orbits in $\mathbb{T}(n)^{0}$ (A.30) and also use of the submersion det $: Q(n) \rightarrow U(1)$ defined by

$$
\operatorname{det}([X]):=\operatorname{det}(X), \quad \forall X \in \mathbb{T}(n)^{0} .
$$

For any $z \in U(1)$, the inverse image $\operatorname{det}^{-1}(z) \subset Q(n)$ consists of the $S(n)$-orbits $[X]$ for which $\operatorname{det}([X])=z$. If $z^{1 / n}$ is an $n$-th root of $z \in U(1)$, then any $[X] \in Q(n)$ 'over $z$ ' is of the form $[X]=\left[z^{1 / n} Y\right]$ for uniquely determined $[Y] \in S Q(n)$. In this way, a choice of $z^{1 / n}$ gives rise to a diffeomorphism between $\operatorname{det}^{-1}(z) \subset Q(n)$ and $S Q(n)$. As a result, we see that

$$
(Q(n), U(1), S Q(n), \operatorname{det})
$$


is a fiber bundle over base $U(1)$ and fiber type given by $S Q(n)$.

Let us cover the unit circle $U(1)$ with two coordinate charts

$$
\begin{aligned}
& \mathcal{U}:=\left\{e^{\mathrm{i} \phi} \mid-\epsilon<\phi<\pi+\epsilon\right\} \simeq(-\epsilon, \pi+\epsilon), \\
& \mathcal{U}^{\prime}:=\left\{e^{\mathrm{i} \phi^{\prime}} \mid-\pi-\epsilon<\phi^{\prime}<\epsilon\right\} \simeq(-\pi-\epsilon, \epsilon),
\end{aligned}
$$

using some small $\epsilon>0$. We can trivialize both $\left.Q(n)\right|_{\mathcal{U}}:=\operatorname{det}^{-1}(\mathcal{U})$ and $\left.Q(n)\right|_{\mathcal{U}^{\prime}}:=\operatorname{det}^{-1}\left(\mathcal{U}^{\prime}\right)$. Working over $\mathcal{U}$ we write any $[X]$ with $\operatorname{det}([X])=e^{\mathrm{i} \phi}$ in the form

$$
[X]=\left[e^{\mathrm{i} \phi / n} Y\right], \quad[Y] \in S Q(n),
$$

and define the trivialization $\chi:\left.Q(n)\right|_{\mathcal{U}} \rightarrow \mathcal{U} \times S Q(n)$ by

$$
\chi:[X] \mapsto(\operatorname{det}([X]),[Y])
$$

Similarly, over $\mathcal{U}^{\prime}$ we can write any $[X]$ with $\operatorname{det}([X])=e^{\mathrm{i} \phi^{\prime}}$ in the form

$$
[X]=\left[e^{\mathrm{i} \phi^{\prime} / n} Y^{\prime}\right], \quad\left[Y^{\prime}\right] \in S Q(n),
$$

and define the trivialization $\chi^{\prime}:\left.Q(n)\right|_{\mathcal{U}^{\prime}} \rightarrow \mathcal{U}^{\prime} \times S Q(n)$ by

$$
\chi^{\prime}:[X] \mapsto\left(\operatorname{det}([X]),\left[Y^{\prime}\right]\right) .
$$

The intersection is the disjoint union $\left.Q(n)\right|_{\mathcal{u} \cap \mathcal{U}^{\prime}}=\left.\left.Q(n)\right|_{\mathcal{V}_{+}} \sqcup Q(n)\right|_{\mathcal{V}_{-}}$, where $\mathcal{V}_{ \pm}$are the connected components of $\mathcal{U} \cap \mathcal{U}^{\prime}$ :

$$
\mathcal{V}_{+}=\left\{e^{\mathrm{i} \phi} \mid-\epsilon<\phi<\epsilon\right\}, \quad \mathcal{V}_{-}=\left\{e^{\mathrm{i} \phi} \mid \pi-\epsilon<\phi<\pi+\epsilon\right\}
$$

We find that on the overlap the two trivializations are related as follows:

$$
\left[Y^{\prime}\right]=[Y] \quad \text { if } \quad \operatorname{det}([X]) \in \mathcal{V}_{+} \quad \text { and } \quad\left[Y^{\prime}\right]=\left[e^{\mathrm{i} 2 \pi / n} Y\right] \quad \text { if } \quad \operatorname{det}([X]) \in \mathcal{V}_{-} .
$$

To be more explicit, we identify the fiber $S Q(n)$ with the open simplex $\operatorname{Simp}_{n-1}$, and then obtain the two coordinate charts

$$
\left.Q(n)\right|_{\mathcal{U}} \simeq \mathcal{U} \times \operatorname{Simp}_{n-1} \simeq\{(\phi, \delta)\} \quad \text { and }\left.\quad Q(n)\right|_{\mathcal{U}^{\prime}} \simeq \mathcal{U}^{\prime} \times \operatorname{Simp}_{n-1} \simeq\left\{\left(\phi^{\prime}, \delta^{\prime}\right)\right\}
$$

The two systems of coordinates coincide on $\left.Q(n)\right|_{\mathcal{V}_{+}}$, and they are related by

$$
\left(\phi^{\prime}, \delta_{1}^{\prime}, \ldots, \delta_{n-2}^{\prime}, \delta_{n-1}^{\prime}\right)=\left(\phi-2 \pi, \delta_{2}, \ldots, \delta_{n-1}, 2 \pi-\sum_{k=1}^{n-1} \delta_{k}\right) \quad \text { on }\left.\quad Q(n)\right|_{\mathcal{V}_{-}} .
$$

Finally, $T^{*} Q(n)$ is covered by the two charts $\left.T^{*} Q(n)\right|_{\mathcal{U}} \simeq T^{*}\left(\mathcal{U} \times \operatorname{Simp}_{n-1}\right)$ and $\left.T^{*} Q(n)\right|_{\mathcal{U}^{\prime}} \simeq$ $T^{*}\left(\mathcal{U}^{\prime} \times \operatorname{Simp}_{n-1}\right)$ with respective canonical coordinates $\left(\phi, p_{\phi}, \delta, \gamma\right)$ and $\left(\phi^{\prime}, p_{\phi^{\prime}}, \delta^{\prime}, \gamma^{\prime}\right)$, for which

$$
\Omega_{\left.T^{*} Q(n)\right|_{\mathcal{U}}}=d p_{\phi} \wedge d \phi+\sum_{j=1}^{n-1} d \gamma_{j} \wedge d \delta_{j}, \quad \Omega_{\left.T^{*} Q(n)\right|_{\mathcal{U}^{\prime}}}=d p_{\phi^{\prime}} \wedge d \phi^{\prime}+\sum_{j=1}^{n-1} d \gamma_{j}^{\prime} \wedge d \delta_{j}^{\prime} .
$$


These two systems of canonical coordinates coincide on $\left.T^{*} Q(n)\right|_{\mathcal{V}_{+}}$and on $\left.T^{*} Q(n)\right|_{\mathcal{V}_{-}}(\underline{\mathrm{A} .43}$ ) their relation is

$$
\left(\phi^{\prime}, p_{\phi^{\prime}}, \delta^{\prime}, \gamma^{\prime}\right)=\left(\phi-2 \pi, p_{\phi}, \delta_{2}, \ldots, \delta_{n-1}, 2 \pi-\sum_{j=1}^{n-1} \delta_{j}, \gamma_{2}-\gamma_{1}, \ldots, \gamma_{n-1}-\gamma_{1},-\gamma_{1}\right)
$$

We finish with two remarks. First, observe from (A.44) that bundle $Q(n)$ (A.37) can be viewed as an associated bundle to a principal $\mathbb{Z}_{n}$-bundle over $U(1)$, with sewing function equal to $1 \in \mathbb{Z}_{n}$ on $\mathcal{V}_{+}$and equal to the constant $e^{\mathrm{i} 2 \pi / n} \in \mathbb{Z}_{n}$ over $\mathcal{V}_{-}$. This principal $\mathbb{Z}_{n}$-bundle is topologically non-trivial. Second, one may check by computing the Jacobian of the coordinate change (A.46) that $Q(n)$ is orientable if and only if $n$ is odd, which was shown previously by a different argument in [21].

Acknowledgements. L.F. thanks J. Balog, C. Klimčík and S.N.M. Ruijsenaars for discussions. This work was supported in part by the Hungarian Scientific Research Fund (OTKA) under the grant K 77400 .

\section{References}

[1] M.A. Olshanetsky and A.M. Perelomov, Classical integrable finite-dimensional systems related to Lie algebras, Phys. Rept. 71, 313-400 (1981)

[2] S.N.M. Ruijsenaars, Systems of Calogero-Moser type, pp. 251-352 in: Proceedings of the 1994 CRM-Banff Summer School 'Particles and Fields', Springer, 1999

[3] F. Calogero, Classical Many-Body Problems Amenable to Exact Treatments, Springer, 2001

[4] B. Sutherland, Beautiful Models, World Scientific, 2004

[5] P. Etingof, Calogero-Moser Systems and Representation Theory, European Mathematical Society, 2007

[6] S.N.M. Ruijsenaars, Action-angle maps and scattering theory for some finite-dimensional integrable systems I. The pure soliton case, Commun. Math. Phys. 115, 127-165 (1988)

[7] S.N.M. Ruijsenaars, Action-angle maps and scattering theory for some finite-dimensional integrable systems II. Solitons, antisolitons and their bound states, Publ. RIMS 30, 8651008 (1994)

[8] S.N.M. Ruijsenaars, Action-angle maps and scattering theory for some finite-dimensional integrable systems III. Sutherland type systems and their duals, Publ. RIMS 31, 247-353 (1995)

[9] S.N.M. Ruijsenaars and H. Schneider, A new class of integrable models and their relation to solitons, Ann. Phys. (N.Y.) 170, 370-405 (1986) 
[10] J.J. Duistermaat and F.A. Grünbaum, Differential equations in the spectral parameter, Commun. Math. Phys. 103, 177-240 (1986)

[11] S.N.M. Ruijsenaars, Finite-dimensional soliton systems, pp. 165-206 in: Integrable and Superintegrable Systems, ed. B. Kupershmidt, World Scientific, 1990

[12] D. Kazhdan, B. Kostant and S. Sternberg, Hamiltonian group actions and dynamical systems of Calogero type, Comm. Pure Appl. Math. XXXI, 481-507 (1978)

[13] A. Gorsky and N. Nekrasov, Relativistic Calogero-Moser model as gauged WZW theory, Nucl. Phys. B 436, 582-608 (1995)

[14] A. Gorsky, Integrable many-body systems in the field theories, Theor. Math. Phys. 103, 681-700 (1995)

[15] G.E. Arutyunov and S.A. Frolov, Quantum Dynamical R-matrices and Quantum Frobenius Group, Commun. Math. Phys. 191, 15-29 (1998)

[16] N. Nekrasov, Infinite-dimensional algebras, many-body systems and gauge theories, pp. 263299 in: Moscow Seminar in Mathematical Physics, AMS Transl. Ser. 2, Vol. 191, 1999

[17] V.V. Fock and A.A. Rosly, Poisson structure on moduli of flat connections on Riemann surfaces and the r-matrix, pp. 67-86 in: Moscow Seminar in Mathematical Physics, AMS Transl. Ser. 2, Vol. 191, 1999

[18] V. Fock, A. Gorsky, N. Nekrasov and V. Rubtsov, Duality in integrable systems and gauge theories, JHEP 07, 028 (2000)

[19] A. Gorsky and A. Mironov, Integrable many-body systems and gauge theories, pp. 33176 in: Integrable Hierarchies and Modern Physical Theories, NATO Science Series II: Mathematics, Physics and Chemistry, Volume 18, eds. H. Aratyn et al., Springer, 2001

[20] L. Fehér and C. Klimčík, On the duality between the hyperbolic Sutherland and the rational Ruijsenaars-Schneider models, J. Phys. A: Math. Theor. 42, 185202 (2009)

[21] L. Fehér and C. Klimčík, Poisson-Lie interpretation of trigonometric Ruijsenaars duality, preprint arXiv:0906.4198, to appear in Commun. Math. Phys.

[22] R. Abraham and J.E. Marsden, Foundations of Mechanics, Second Edition, AddisonWesley, 1978

[23] V.V. Prasolov, Problems and Theorems in Linear Algebra, AMS, 1994

[24] J.F. van Diejen and L. Vinet, The quantum dynamics of the compactified trigonometric Ruijsenaars-Schneider model, Commun. Math. Phys. 197, 33-74 (1998)

[25] A. Alekseev, A. Malkin and E. Meinrenken, Lie Group Valued Moment Maps, J. Diff. Geom. 48, 445-495 (1998)

[26] A.A. Oblomkov, Double affine Hecke algebras and Calogero-Moser spaces, Rep. Theor. 8, 243266 (2004) 